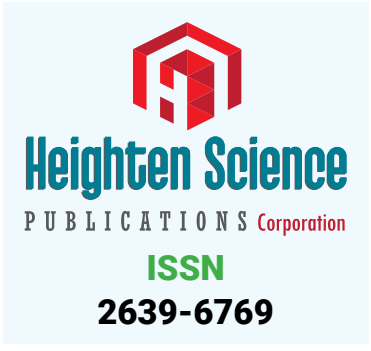

*Address for Correspondence: Luisetto $\mathrm{M}$, Applied Pharmacologist Independent Researcher, 29121, Italy, Email: maurolu65@gmail.com Submitted: 24 May 2018
Approved: 18 June 2018
Published: 19 June 2018

Copyright: @ 2018 Luisetto M, et al. This is an open access article distributed under the Creative Commons Attribution License, which permits unrestricted use, distribution, and reproduction in any medium, provided the original work is properly cited.

Keywords: Organotropism; Liver metastasis; Cancer; Pathology, Immune systems; New therapeutic strategies; Biology; Botany; Cancer; Metastasis; Organ specific metastasis; Metastatic colonization
Research Article

\section{Similarity between Some Biological Systems, Organotropism and Metastatic Process: Active Role Played By Secondary Organ?}

\author{
Luisetto $\mathrm{M}^{1 *}$, Behzad Nili-Ahmadabadi ${ }^{2}$, Hossein Nili- \\ Ahmadabadi ${ }^{3}$, Ghulam Rasool Mashori ${ }^{4}$, Gamal Abdul Hamid ${ }^{5}$, \\ Kausar Rehman Khan ${ }^{6}$, Behrooz Nili-Ahmadabadi ${ }^{7}$, Ahmed \\ Yesvi Rafa ${ }^{8}$ and Luca Cabianca ${ }^{9}$ \\ ${ }^{1}$ Applied Pharmacologist Independent Researcher, 29121, Italy \\ ${ }^{2}$ Nano Drug Delivery (A Product Development Firm), Chapel Hill NC, USA \\ ${ }^{3}$ Yasuj University of Medical Sciences, Faculty of Medicine, Yasuj, Iran \\ ${ }^{4}$ Department of Medical \& Health Sciences for Woman, Peoples University of Medical and \\ HealthSciences for Women, Pakistan \\ ${ }^{5} \mathrm{Hematology}$ Oncology, University of Aden, Aden, Yemen \\ ${ }^{6}$ Preston University Karachi, Pakistan \\ ${ }^{7}$ Animal Biology, Scientist at Mindcracy \& Nano4Rx \\ ${ }^{8}$ Founder and President, Yugen Research Organization (YRO), Independent Researcher \\ Bangladesh \\ ${ }^{9}$ Biomedical Laboratory, Citta Della Salute Turin, Italy
}

\section{Abstract}

According to literature, about $90 \%$ of death from cancer is related to metastasis. Metastatic process present many similarity to some other biological processes. Once we have examined some relevant biomedical literature, by understanding the real causes of metastasis, it would become much more possible to introduce new therapeutic strategies to delay or in some cases even to stop this kind of killer process. Breast cancer, as an example, produces metastasis to different organs, which seems to be related to the subtype. We believe that a deep understanding of the roles of breast cancer cells and their interactions with the liver microenvironment in early breast cancer metastasis could be a crucial factor for the design and development of effective BCLM breast cancer liver metastases therapeutic strategies and to better understand the general process. Let's suppose the secondary organ or organs can be considered as incubator/s for the primary metastatic cells. What kind of consequences we can have in therapy field if there is an active regulating role in determining the location of secondary cancers?

Let's observe the role played by liver, bone marrow, CNS central nervous system, lungs, lymphocytes and other secondary locations/organs a little bit closer or maybe from a different angle let's suppose we try to come up with just a hypothesis. Just let's take this as a possibility, and we take the thread to see where it takes us.

\section{Introduction}

Cancer has been a big puzzle in medicine so much that the oncologists kneeled down in front of cancer cells by confessing that one dumb cancerous cell is more intelligent than 1,000 smart scientists. So is it a really a troubleshoot problem or maybe it is that we still don't know what cancer is, so basically it is a science problem. Observing the global efficacy in some cancer disease we can say that not all neoplasia are actually well treated as request. (In order to achieve high clinical results in fact in many cases patients present exitus even pharmacologically treated with current therapy). 
In many situations this is related to the metastatic process in secondary organs. For this reason is necessary verify better the metastatic process in order to think to new therapeutic strategies that can gives better clinical results. The same observing the clinical advantages related to some new MABS introduced in therapies with few month in patient surviving and officially registered by governative health authority.

Even if today some cancer are efficacy treated in other kind of cancer disease something else can be do. IN 1889 S. Paget proposed his "seed and soil" theory of cancer. He analyzed more than 1000 autopsy records of women who had breast cancer and found that the patterns of metastasis were not a random process :according this theory tumor cells (the seeds) have a specific affinity for specific tissue - organs (the soil), and metastasis would only result if the seed and soil were compatible. But this famous theory can be improved?

It's not a simple seed and soil means filtration that might determine the secondary organ, but it's a competition that could include the active role played by the secondary organ and:

1. The presence of bindings- interactions ( in an active incubation organ) or maybe

2. The fusion of cells with parenchymal with undifferentiated properties such as bone marrow, and liver and also

3. The secondary organ features could play an important role such as a. high the blood irrigation,

b. the availability of raw material for cell growth( biochemical molecule, energy, oxygen)

4. The role of growth factors in malignating,

5. And many other factors.

MABS monoclonals antibodies currently in use in example recognize some growth factor receptors and show much clinical efficiency.

But even associated with chemiotherapy and other (as radiotherapy or surgery) not efficacy prevent all kind metastatic process in useful way. Can we identify other growth factor or other molecule deeply involved in this active role by secondary organ?

Can we think to control the active role of liver in example to prevent metastatic disease?

So let's have a more independent thinker free mind, skeptical look at the entire process, maybe we are going in the wrong direction all along or we don't have the right view at the problem. We are aught to believe that the migration of a primary cancerous cell is merely a physical filtration process in which the detached metastatic cell, unleashed into the blood stream, would be eventually entrapped by the capillaries of one of the organs of the reticulum endothelial system, and that's where the primary cancer is having a second, third nest or fourth nest. If that is the fact, then there shouldn't be that much difference except for the size of the detached cell from the original cancerous primary site, whether it is hepatoma, prostate or colon cancer. The fact is that the primary tumoral cell is migrating to a secondary organ, and all blood vessel, the veins and linphatic way, (in example through the portal vein into the liver, and from there distribute to other organs), including lung, lymph nodes, spleen, bone marrow, brain et other.

But that's not so simple: We see that certain primary cancers have the tendency to migrate to specific organs, different than other types of primary cancers, and it doesn't 
seem to be a size exclusion principle governing that process. It seems that the filtration narrative is an oversimplification. It seems that there should be some sort of hosting tissue in which the primary cell is first stationed, and then the migration process, right there, in what we call it the incubator organ, is governed by an intelligent active interaction between the primary cancer cells and harboring sanctuary incubation tissue, in our believe maybe liver or bone marrow, but these are just wild guesses would believe maybe other organs could also act as the incubation sites.

There are good reasons to our believe the incubation sites must have certain reproductive, blast cell type or what-you-call-it stem cell properties. Of course only when we find the right mechanism, then it will be possible to find new efficient therapeutic strategies to at least delay, if not completely stop the metastatic diffusion of some types of cancer. Our goal in this paper is to analyze some natural processes or mechanisms used by cells to direct certain cells towards some distant tissues and organ. This preferential choice is responsible of dangerous metatastic diffusion in some kinds of cancer patients.

Have you ever thought why some bacterial or viral infections cause meningitis? What kind of factors play the role for microorganism to become neurotropic?

Why in KURU disease, the prion causes a specific central nervous system disease come on with the Port of Entry is the digestive tract? What makes a microorganism to specifically pick certain organ on others? [1]. Well another example you could mention is MICORRIZZA phenomena: fungal and superior plants through a mutual benefit exchange (symbiotic association) with mutual benefits: metabolic and other [2]. We said we take different biological especially human biological models to see if we can learn from these models, and find out they may be using like model to explain mechanisms for metastasis. Fetus, being very similar to tumoral cells as far as differentiation, is the something that comes to my mine. So in embryology the polarization and migration are fundamental facts in development of embrions (Segmentation, polarized migration) [3]. I'm sure you can see where this is going. So in the development a fetus, we can see similar velocity cell growth very similar to that of for example neoplastic cells (and same increase in cellularity)

What time also see a similar relationship in in the endo-symbiotic theory of organelles such as mitochondria. This Theory says that mitochondria originated from little prokaryotic self that formed hosts in larger prokaryotic cells. When the atmosphere changed, which was accompanied with an increase in oxygen level, some aerobia cells protested parassitate anaerobia cells, transitioning from ananaerobic to efficient form of life namely aerobic metabolism. So the question that just comes to everyone's mind is why or how this colonization for a better metabolic/energetic outcome have occurred?

From A TELEGRAPH ARTICLE: Life existed in the oceans 200 million years before oxygen appearerd on Earth: "life evolved at least 200 million years before oxygen began to build up in the atmosphere, a study has shown. During this period in its history, known as the Archaean, the Earth was was covered by a poisonous smog of methane, ammonia and other toxic gases. Similar conditions exist today on Saturn's moon Titan. Life as we know it today could not have survived on the early Earth. The new study involved an analysis of ancient preserved seabed rocks from South Africa dating back two to three billion years.

US scientists at Rutgers University in New Brunswick, New Jersey, found chemical evidence of nitrogen cycles that could not have taken place without the presence of free oxygen. Nitrogen cycles relate to the way living beings cane obtain and nitrogen use to produce complex organic molecules.

Evidence of nitrogen cycles provides a "fingerprint" of life. 
The researchers, Dr Linda Godfrey and Dr Paul Falkowski, concluded that organisms which produced oxygen as a by-product of photosynthesis must have evolved by around 2.5 billion years ago. Oxygen did not begin to enrich the atmosphere until at least 200 million years later. The scientists wrote in the journal Nature Geoscience: "Nitrogen is a relatively inert molecule and has an atmospheric lifetime of the order of around one billion years. In contrast, oxygen is highly reactive and must be produced continuously by oxygenic photosynthesis."

"It is unlikely that the gas was present above trace levels in the atmosphere of the Earth during the first two billion years of the planet's history, but when oxygenic photosynthesis first arose on the Earth is not known with certainty." In non-equilibrate system energy will global reduce inside. (Potential and chemical energy). We can think to a simple experiment to better explain the concept: 2 laboratory becker filled up whit 2 different media (different composition in nutrient). If connected by a duct and added some kind of cell that can move themselves, the question is in what direction they will go? Would they prefer the ritcher culture media? Or not? And if we fill up this 2 different beakers with different tissue extracts what would happen then?

Even if breast metastasis involved the liver due by circulation preferences 2 lymphatic or other organs through physical entrapment process why we have high levels of certain organs vs others? Could it be that this secondary organ offers some sort of growth factors to incubate this cell? Or it is that inside the hepatocytes, the microenvironment is more fovoreable than other organs? Fetus liver is a hemopoietic organ and this show the high function that this organ can play (related to the phenotypic expression time related).

How come in critical diabetic patients for example, pancreatic langerhans Isles how are currently transplanted in the liver? Could it be that liver have some sort of stem-cell properties? Could it be that hepatocytes could fuse with other cells, and differentiate to some other types of cells with specific functions? Many biological systems would find other mechanisms to reach the natural equilibrium or balance themselves in more efficient ways and providing advantages to different populations in the same environment or even ecosystem.

Other example can be embryo implant in utero (active function in embryo- fetal development). So the question is if uterus can host the fetus of another man, could this model also be applied to hepatocytes for malignant cancer? New pharmacological strategies to avoid or delay invasion, implants and other system could be introduced by interact with the incubator role of secondary organ.

\section{Materials and Methods}

First whit a review method observing some, in our opinion, relevant biomedical literature we try to produce new theories usefull in metastasis process control. Then we try to submit an experimental project to verify some metatastic cell specific migrating properties and role played by secondary organ to support cell growth condition. The biomedical literature was obtained using scientific database (PUBMED). Adding the observational methods (some relevant in our opinion biomedical literature) to an experimental theoretical project we can obtain new knowledge in metatstatic process.

\section{Results of the Observational Review}

\section{According relavant biomedical literature}

- $\quad$ Mark C. Brundrett: “. The first bryophyte-like land plants, in the early Devonian (400 million years ago), had endophytic associations resembling vesicular-arbuscular mycorrhizas (VAM) even before roots evolved. Mycorrhizal evolution would have progressed from endophytic hyphae towards balanced associations where partners were interdependent due to the exchange of limiting energy and nutrient resources. 
Most mycorrhizas are mutualistic, but in some cases the trend for increasing plant control of fungi culminates in the exploitative mycorrhizas of achlorophyllous, mycoheterotrophic plants. Ectomycorrhizal, ericoid and orchid mycorrhizas, as well as nonmycorrhizal roots, evolved during the period of rapid angiosperm radiation in the Cretaceous. It is hypothesised that roots gradually evolved from rhizomes to provide more suitable habitats for mycorrhizal fungi and provide plants with complex branching and leaves with water and nutrients. Selection pressures have caused the morphological divergence of roots with different types of mycorrizas [2] ".

- Daniel S. Heckman et al writed that: "The colonization of land by eukaryotes probably was facilitated by a partnership (symbiosis) between a photosynthesizing organism (phototroph) and a fungus. Our protein sequence analyses indicate that green algae and major lineages of fungi were present $1000 \mathrm{Ma}$ and that land plants appeared by $700 \mathrm{Ma}$, possibly affecting Earth's atmosphere, climate, and evolution of animals in the Precambrian" [4].

- Vogel DY et al "In neuroinflammatory diseases, macrophages can play a dual role in the process of tissue damage, depending on their activation status (M1 / M2). M1 macrophages are considered to exert damaging effects to neurons, whereas M2 macrophages are reported to aid regeneration and repair of neurons. Their migration within the central nervous system may be of critical importance in the final outcome of neurodegeneration in neuroinflammatory diseases e.g. multiple sclerosis (MS). To provide insight into this process, we examined the migratory capacity of human monocyte-derived M1 and M2 polarised macrophages towards chemoattractants, relevant for neuroinflammatory diseases like MS.Primary cultures of human monocytederived macrophages were exposed to interferon gamma and lipopolysaccharide (LPS) to evoke proinflammatory (M1) activation or IL-4 to evoke anti-inflammatory (M2) activation. In a TAXIS can assay, migration of M0, M1 and M2 towards chemoattractants was measured and quantified. Furthermore the adhesion capacity and the expression levels of integrins as well as chemokine receptors of M0, M1 and M2 were assessed. Alterations in cell morphology were analysed using fluorescent labelling of the cytoskeleton. Significant differences were observed between M1 and M2 macrophages in the migration towards chemoattractants. We show that M2 macrophages migrated over longer distances towards CCL2, CCL5, CXCL10, CXCL12 and C1q compared to nonactivated (M0) and M1 macrophages. No differences were observed in the adhesion of M0, M1 and M2 macrophages to multiple matrix components, nor in the expression of integrins and chemokine receptors.

Our results indicate that the alternative activation status of macrophages promotes their migratory properties to chemoattractants relevant for neuroinflammatory diseases like MS. Activated, proinflammatory macrophages have reduced migratory properties. Based on our results, we postulate that the activation status of the macrophage influences the capacity of the macrophages to rearrange their cytoskeleton. This is the first step in understanding how modulation of macrophage activation affects macrophage migration in neuroinflammatory diseases like MS [5] ".

- $\quad$ Nance J writed that :."Polarization of early embryos along cell contact patterns-referred to in this paper as radial polarization--provides a foundation for the initial cell fate decisions and morphogenetic movements of embryogenesis. Although polarity can be established through distinct upstream mechanisms in Caenorhabditis elegans, Xenopus laevis, and mouse embryos, in each species, it results in the restriction of PAR polarity proteins to contact-free surfaces of blastomeres. In turn, PAR proteins influence cell fates by affecting signaling pathways, such as Hippo and Wnt, and regulate morphogenetic movements by directing cytoskeletal asymmetries [3]".

- $\quad$ According William F. Martin et al: "For over 100 years, endosymbiotic theories have figured in thoughts about the differences between prokaryotic and eukaryotic 
cells. More than 20 different versions of endosymbiotic theory have been presented in the literature to explain the origin of eukaryotes and their mitochondria. Very few of those models account for eukaryotic anaerobes. The role of energy and the energetic constraints that prokaryotic cell organization placed on evolutionary innovation in cell history has recently come to bear on endosymbiotic theory. Only cells that possessed mitochondria had the bioenergetic means to attain eukaryotic cell complexity, which is why there are no true intermediates in the prokaryote-to-eukaryote transition. Current versions of endosymbiotic theory have it that the host was an archaeon (an archaebacterium), not a eukaryote. Hence the evolutionary history and biology of archaea increasingly comes to bear on eukaryotic origins, more than ever before. Here, we have compiled a survey of endosymbiotic theories for the origin of eukaryotes and mitochondria, and for the origin of the eukaryotic nucleus, summarizing the essentials of each and contrasting some of their predictions to the observations. A new aspect of endosymbiosis in eukaryote evolution comes into focus from these considerations: the host for the origin of plastids was a facultative anaerobe" [6].

In bone marrow transplant procedure staminal cell injectes colonize bone marrow.

- $\quad$ Francesco Fiz et al: "Mechanisms of hematopoietic reconstitution after bone marrow (BM) transplantation remain largely unknown. We applied a computational quantification software application to hybrid FDG PET/CT images to assess activity and distribution of the hematopoietic system throughout the whole skeleton of recently transplanted patients. 34 patients underwent PET/CT 30 days after either adult stem cells (ACT, $n=18$ ) or cord blood transplantation (CBT, $n=16$ ). Our software automatically recognized compact (CBV) and trabecular bone (IBV) in CT slices. Within IBV, co-registered PET data were extracted to identify the active BM (ABM) from the inactive tissue. Patients were compared with 34 matched controls, chosen among a published normalcy database. Whole body ABM increased in ACT and CBT when compared to controls $(12.4 \pm 3$ and $12.8 \pm 6.8 \mathrm{vs} 8.1 \pm 2.6 \mathrm{ml} / \mathrm{Kg}$ of ideal body weight, IBW, $\mathrm{p}<0.001)$. In long bones, ABM was three- and six-fold increase in CBT and ACT, respectively, in comparison with controls $(0.9 \pm 0.9$ and $1.7 \pm 2.5$ vs. $0.3 \pm 0.3 \mathrm{ml} / \mathrm{Kg} \mathrm{IBW}$, $\mathrm{p}<0.01)$. These data document an unexpected distribution of transplanted BM into previously abandoned bone marrow sites" [7].

- $\quad$ Rui Ma et al.: "the formation of liver metastases is not random. Indeed, breast cancer cell factors contribute to the liver microenvironment. The process of liver metastasis consists of multiple steps and involves various factors from breast cancer cells and the liver microenvironment. Breast cancer is the leading cause of cancerrelated deaths in female patients worldwide. (Five subtype: luminal A, luminal B, human epithelial growth receptor type 2 (HER-2), basal-like, and claudin-low) the 5 -year survival rate for primary breast cancer is $99 \%$. One-third of breast cancer patients will present with distant non-nodal metastases, and the 5-year survival rate decreases to $23 \%$ once distant metastases have developed.

Breast cancer mainly metastasizes to the bony skeleton, lungs, liver, and brain via the circulation; the liver is a common metastatic site for solid cancers and represents the third most common site for breast cancer. If breast cancer liver metastasis (BCLM) is left untreated, the survival time is 4-8 months. The formation and growth of breast cancer cells in the liver is a complex process. The most accepted model for metastasis is the "seed and soil" hypothesis (by S. Paget in 1889).: Organ metastases form merely when the seed (disseminated tumor cells) and soil (secondary organ) are compatible Semenza separated the process of blood vessel metastasis of breast cancer into the following steps: intravasation, circulation, margination, extravasation, and colonization; The hepatic microenvironment - liver sinusoidal structure is real crucial for the initial arrest of breast cancer and progression within the liver. 
A deeply understanding of the roles of breast cancer cells and the liver microenvironment in early breast cancer metastasis is fundamental for the development of effective BCLM therapies. Studies showed that the inflammatory response correlates with the liver metastatic potential of some tumors. The inflammatory factor, TNF- $\alpha$, can trigger the expression of E-selectin in endothelial cells, including liver sinusoidal endothelium cells. Breast cancer cells have the ability to initiate an inflammatory cascade, which increases adhesiveness to liver sinusoidal endothelium cells, similar to that induced by colorectal and lung cancers. The process of tumor cell attachment to the endothelium during metastasis is multifactorial, the production of TNF- $\alpha$-induced endothelial E-selectin in tumor cells appears to be a key step in the BCLM process. Asgeirsson et al. Reported that induction of IL-6 decreased cell adhesion in three breast cancer cell lines, and was associated with reduced E-cadherin expression. Patients with breast cancer liver metastases had significantly higher IL-6 levels. It appears that breast cancer cells create a pro-inflammatory microenvironment which triggers adhesion and invasion of tumor cells into the liver by secreting a number of cytokines.

Breast cancer cells express many chemokine receptors that initiate liver metastasis, C-X-C Chemokine Receptor type 4 (CXCR4) is the most common. The ligand of CXCR4, stromal-derived factor 1- $\alpha$ (SDF-1, CXCL12), is also highly expressed in the liver, indicating that the CXCL12/CXCR4 interaction might contribute to BCLM. CXCR4 plays an important role in modulating breast-to-liver metastasis through integrin-adhesionreceptor signaling. The availability of chemokine receptors on tumor cell surfaces, the presence of specific ligands within the microenvironment of potential target organs, and the suitability of the extracellular matrix (ECM) composition appear to be required for successful extravasation of breast cancer cells in the early metastatic process.

In tumor-bearing mice, $\mathrm{CC}$ chemokine ligand 2 (CCL2) neutralizing antibodies inhibit the growth and liver metastases of primary breast cancer by reducing cell proliferation, survival, and tumor-associated macrophage (TAM) recruitment. Induced mesenchymal stromal cells (MSCs) produce CCL5, and significantly promote breast cancer cell migration to the liver in vivo and in vitro. Chemokine receptors can enhance breast cancer metastasis to the liver. Porter et al. E-cadherin expression in liver metastatic sites is due to loss of promoter methylation. Breast cancer cells that re-express E-cadherin revert back to an epithelial phenotype. The epithelial-to-mesenchymal transition (EMT) of cancer cells contributes to increased invasion and dissemination.

IN the metastatic site, a mesenchymal-to-epithelial transition (MET) occurs, leading to the colonization and growth of metastatic foci. Expression of the cell adhesion molecule, E-cadherin, in breast cancer cells can facilitate breast cancer cell adhesion to hepatocytes for seeding in the liver. Breastcancer cells that express E-cadherin are able to form liver metastases. Chao et al. Showed that the liver microenvironment can induce breast cancer cells to reexpress E-cadherin and cause MET. This phenotypic change has the potential to alter cell behavior, and thus may be a critical step for cells to survive at metastatic sites within the liver.

Re-expression of E-cadherin, accompanied by a partial MET in the liver, increases post-extravasation survival of metastatic cancer cells and may help to elucidate why chemotherapy commonly fails to treat the BCLM. N-cadherin activates a metastatic signaling pathway coordinated by fibroblast growth factor receptor (FGFR) and matrix metalloproteinase-9 (MMP-9) to overcome the suppressive effects regulated by E-cadherin. Receptors of the integrins family are important components of the ECM. Integrin complexes regulate adhesion, apoptosis, proliferation, differentiation, migration, invasion, and metastasis.

Ig-SF: Epitheli1al cell adhesion molecule (EpCAM/CD326), a transmembrane protein, plays a variety of roles in cell proliferation, adhesion, migration, and tissue maintenance. EpCAM is overexpressed in many cancer, and also in breast cancer; a recent study suggested that $\mathrm{p} 53$ dysfunction may explain this phenomenon. In patients 
with node-positive primary breast cancer, elevated EpCAM expression correlates with diminished overall survival, overexpression of EpCAM promotes cancer progression and metastasis. Cancer stem cells (CSCs) within tumors have cancer-initiating potential and metastatic capability. High levels of CD44 expressed by CSCs are believed to be involved in adhesion, invasion, apoptosis resistance, and metastasis. Breast cancer cells expressing high levels of CD44 and low CD24 maintain stemness properties it appears that cell adhesion molecules play important roles in BCLM Role.

Cadherins: The up-regulation of claudin- 3 and -4 is correlated with poor prognosis and the breast cancer basal-like subtype. The loss of claudin- 4 and -7 promoted liver metastasis of breast cancer cells in Balb-c mice. Five major subtypes of breast cancer have different abilities to metastasize to distant organs, and share pathways with the preferred metastatic sites. Patients with bone relapses have the luminal subtypes of breast cancer most frequently. The HER-2 subtype may metastasize to bone via processes that differ from the luminal subt. The basal subtype often metastasizes to the brain and lungs.

Rodriguez-Pinilla et al.: The basal-like subtype metastasizes more frequently to the lungs and other visceral organs, brain and liver, but no bone metastases were detected. Studies in patients have suggested a high incidence of brain metastases arising from basal-like tumors (ER-/PR-/HER2-, and usually identified as "triple-negative" breast cancer [TNBC]), but it has been reported that p53-negative TNBC has an increased tendency to develop lung metastases. Duan et al. showed that the breast cancer subtype is an independent prognostic predictor for patients with breast cancer metastases to the liver. Survival after liver metastases arising from TNBC is 21 months compared to 30,32 , and 41 months for patients with the HER-2, luminal B, and luminal a subtypes; liver metastases from TNBC has the worst prognosis.

Factors associated with the liver microenvironment: Hypoxia-inducible factorregulated genes. Hypoxia-inducible factors (HIFs) activate the transcription of target genes that are involved in many aspects of breast cancer progression, such angiogenesis, metabolic reprogramming, local tissue invasion, and metastasis. HIFs not only activate lysyl oxidase (LOX) directly to inhibit liver metastases, but osteopontin (OPN), vascular endothelial growth factor (VEGF), and TWIST promote BCLM. It has been suggested that some of the hypoxia-inducible factor-regulated genes contribute to BCLM. LOX is an amine oxidase that contributes to the formation of ECM. Secreted by fibrinogenic cells (residues in collagen and elastin to maintain the structural stabilization of ECM).

Erler et al.: hypoxia-induced LOX has a key function in the metastasis of breast cancer cells. Erler et al.: significant effects on growth in metastatic sites within the liver. The effects of LOX on cell adhesion, migration, invasion, and three-dimensional growth are more crucial for liver metastatic growth than primary breast cancers. HIFinduced OPN is a secreted phosphoprotein functioning as a cell attachment protein by binding two cell adhesion molecules ( $\alpha \mathrm{v} \beta 3$ integrin and CD44). OPN is overexpressed in tumors and elevated serum OPN levels are associated with advanced metastatic cancer. Gain- and loss-of-function assays have demonstrated the critical role for OPN in tumor metastases. Cancer cells express an angiogenic factors. VEGFs (neovascularization). Inhibition of VEGFSs signals has been shown to suppress breast cancer progression and lung metastases.

Chien et al.: Inhibition of VEGFR/FGFR kinases drastically reduce the formation of liver metastases and decreased primary breast cancer growth. TWIST is a basic helixloop-helix transcription factor. TWIST mainly regulates gastrulation and mesoderm specification. Recently, TWIST has been shown to play an important role in mediating cancer metastasis. TWIST is a downstream target of HIF- 1 and has role in metastatic phenotypes induced by hypoxia or overexpression of HIF-1 $\alpha$ in breast cancer cell lines (MCF-7). HIF-1 $\alpha$ promotes hypoxia-induced breast cancer progression -metastasis 
through the direct activation of TWIST expression. Role of the vasculature might be essential for liver metastasis.

Vermeulen et al.: colorectal cancer metastases to the liver grow according to three different histologic patterns, termed 'pushing', 'replacement', and 'desmoplastic' growth patterns. The results of studies involving liver metastases of colorectal and breast cancers showed that the different grow patterns have different angiogenic properties. The pushing and desmoplastic patterns grow in an angiogenic fashion, which is at least in part hypoxia-driven.

Martin et al.: the majority of early metastatic foci in the liver contain few cells, even 12 days after breast cancer cell injection. Only a few foci were able to develop into micrometastatic lesions with a patent vasculature, so lesions that utilize an existent patent blood supply can thrive in the liver microenvironment, while the remaining foci without a vascular supply remain dormant in the liver.

Naumov et al. : Tumors are dependent on angiogenesis for progressive growth and remain harmless to the organism at the non-angiogenic dormant stage. The expansion of tumor mass is associated with recruiting endothelial cells after the cancer tissues undergo a switch from a non-angiogenic dormant phenotype to the angiogenic phenotype. Studies have reported that the initial arrest of cancer cells in the sinusoids of the liver is restricted by the sizes of cancer cells. Haier et al. : Tumor cells adhere to sinusoidal capillaries, the internal diameter of which is larger than the tumor cells. Unique structural features of liver, including the existence of a fenestrated endothelium and lack of an organized sub-endothelial basement membrane, have a HIGH impact on the interactions between breast cancer cells and the liver microenvironment.

The fenestrated endothelium controls liver-specific microvascular exchange and impacts the ability of cells to transmigrate through the vessels into the microenvironment of liver. Breast cancer cells extend cellular projections through the fenestrated endothelium into the space of Disse on seeding the liver, which makes direct contact with hepatocytes.

Martin et al.: breast cancer cells are bound to vessels with clear vascular labeling in the sites of liver metastases. Sinusoidal capillaries play a significant role in the initial arrest of breast cancer. Koo et al. verify the status of ER, PR, and HER-2 in primary and metastatic breast cancers and determined the relationship between ER, PR, and HER-2 and organ-specific metastases of breast cancer. ER+ or PR+/HER-2- (luminal A) subtypes were predominant in the sites of liver metastases (75.0\%). Increased phosphorylation of HER-2 appears to be very important for the establishment of breast cancer liver metastases.

So A new model for breast cancer liver metastasis, as follows: intravasation: invasive breast cancer cells invade via the endothelium of a tumor blood vessel into the circulation; circulation: breast cancer cells survive in the blood vessels and lack of cell-cell or cell-matrix attachments; margination: CTCs arrest at the liver site by adhering to the sinusoidal endothelial cell via specific sets of adhesion molecules, such as cadherins, integrins, Ig-SF, and CD44; extravasation: the migrated breast cancer cells invade through the endothelial wall of sinusoidal endothelial cells, migrates, and finally proliferates in the liver (in this process, the diameter of the sinusoidal endothelium and the lack of an organized sub-endothelial basement membrane have a great impact on breast cancer cell migration); and colonization: breast cancer cells survive and form a life-threatening macrometastatic focus in the liver microenvironment by mediating hypoxia-inducible factor-regulated genes (LOX, OPN, VEGF, and TWIST), the status of ER, PR, and HER-2 expression, and angiogenesis for breast cancer cells" [8].

- Kawada $\mathrm{K}$ et al.: Colorectal cancer is the second most common cancer, and is the third leading cause of cancer-related death in Japan. The majority of these deaths is attributable to liver metastasis. Recent studies have provided increasing 
evidence that the chemokine-chemokine receptor system is a potential mechanism of tumor metastasis via multiple complementary actions: (a) by promoting cancer cell migration, invasion, survival and angiogenesis; and (b) by recruiting distal stromal cells (i.e., myeloid bone marrow-derived cells) to indirectly facilitate tumor invasion and metastasis. Here, we discuss recent preclinical and clinical data supporting the view that chemokine pathways are potential therapeutic targets for liver metastasis of colorectal cancer [9].

- $\quad$ Chirag S et al.: "The liver has been the site of choice for pancreatic islet transplantation in clinical practice" [10].

Maxim Norkina et al.: "Hematopoietic cell transplantation (HCT), once used as a last-resort therapy, is now considered a lifesaving procedure for thousands of patients with life-threatening diseases worldwide and is frequently used early in the course of treatment for diseases destined to be uncontrollable by non-HCT therapies" [11].

- Vogel DY et al.: "In neuroinflammatory diseases, macrophages can play a dual role in the process of tissue damage, depending on their activation status (M1 / M2). M1 macrophages are considered to exert damaging effects to neurons, whereas M2 macrophages are reported to aid regeneration and repair of neurons. Their migration within the central nervous system may be of critical importance in the final outcome of neurodegeneration in neuroinflammatory diseases e.g. multiple sclerosis (MS).

Primary cultures of human monocyte-derived macrophages were exposed to interferon gamma and lipopolysaccharide (LPS) to evoke proinflammatory (M1) activation or IL-4 to evoke anti-inflammatory (M2) activation. In a TAXIScan assay, migration of M0, M1 and M2 towards chemoattractants was measured and quantified. Furthermore the adhesion capacity and the expression levels of integrins as well as chemokine receptors of M0, M1 and M2 were assessed. Alterations in cell morphology were analysed using fluorescent labelling of the cytoskeleton. Significant differences were observed between M1 and M2 macrophages in the migration towards chemoattractants.

Significant changes were observed in the cytoskeleton organization upon stimulation with CCL2, M0, M1 and M2 macrophages adopta spherical morphology and the cytoskeleton is rapidly rearranged. Together our results indicate that the alternative activation status of macrophages promotes their migratory properties to chemoattractants relevant for neuroinflammatory diseases like MS. The activation status of the macrophage influences the capacity of the macrophages to rearrange their cytoskeleton" [5].

- $\quad$ According Paweletz $\mathrm{N}$ et al.: "Analyzing the different steps of malignant growth (primary tumor, metastasizing tumor cells, secondary tumor), one recognizes an intense interaction between normal and malignant cells. Tumor cells not only induce activities of normal cells, which normally are rarely activated, but also they exploit properties of normal cells for their own purposes. The major mechanisms and processes of this "parasitism" are described in more detail and the results are discussed. Tumors cannot grow beyond a certain size without a supply of blood and lymph vessels by the host (angiogenesis). Metastasizing tumor cells cannot leave the vessel (extravasate) in which they are transported without the cooperation of the respective endothelial cells of the host. An appropriate environment formed by the host tissues is essential for the settlement of tumor cells at secondary sites" [12].

- A C Selden et al.: in "Growth factors and the liver: The capacity of the liver to regenerate after severe viral or drug induced hepatitis, or after massive partial hepatectomy, is remarkable. Experimentally, is reported regeneration after the performance of a $70 \%$ partial hepatectomy in rats, in whichnormal liver mass is restored within 10 days" [13]. 
- Yachao Tao et al.: "Liver regeneration is a highly organized tissue regrowth process and is the most important reaction of the liver to injury. The overall process of liver regeneration includes three phases: priming stage, proliferative phase, and termination phase. The initial step aims to induce hepatocytes to be sensitive to growth factors with the aid of some cytokines, including TNF- $\alpha$ and IL-6. The proliferation phase promotes hepatocytes to re-enter G1 with the stimulation of growth factors. While during the termination stage, hepatocytes will discontinue to proliferate to maintain normal liver mass and function. Except for cytokine- and growth factormediated pathways involved in regulating liver regeneration, new substances and technologies emerge to influence the regenerative process. Here, we reviewed novel and important signaling molecules involved in the process of liver regeneration to provide a cue for further research" [14].

- $\quad$ "The liver, composed of parenchymal cells-hepatocytes-and nonparenchymal cells including endothelial cells, Kupffer cells, lymphocytes, and stellate cells, has a unique capacity to precisely regulate its growth and mass, which is particularly remarkable since hepatocytes are stable cells and rarely divide in the normal state, as they are quiescent in the G0 phase of the cell cycle. However, their proliferative capacity is initiated in the case of liver tissue loss. There are two different regenerative models. Partial hepatectomy (two-thirds of the liver is removed) initiates a unique response, during which the remaining diploid hepatocytes enter into the cell cycle to compensate for the loss of liver tissue, taking about a week. Another pattern of the regenerative model is established by insult, such as toxins and viral infection, during which all hepatocytes are hurt and oval cells are considered as potent stem cells to differentiate into hepatocytes and biliary cells. Both of the two patterns of liver regeneration will be involved in the review.

Liver regeneration is a complex network regulated by various growth factors and cytokines expressed at the site of injury or migrated to the liver via the circulatory system. To sum it up, the regenerative process includes three critical steps [3]: firstly, quiescent hepatocytes convert from G0 to G1 of the cell cycle when faced with multiple stimulations (the priming phase); secondly, with the help of mitogens, hepatocytes progress beyond the restriction point to the G1 phase and then the mitosis (the proliferation phase); and then the last, cells terminate proliferation under the control of negative factors (the termination phase), such as transforming growth factor beta (TGF- $\beta$ ) and activin. In these three phases, various cytokines or growth factors exhibit a pivotal role through cell signaling pathways of multiple biological effects" [14].

- $\quad$ Taylor DP et al.: writed that: "Nearly half of breast carcinoma metastases will become clinically evident five or more years after primary tumor ablation. This implies that metastatic cancer cells survived over an extended timeframe without emerging as detectable nodules. The liver is a common metastatic destination, whose parenchymal hepatocytes have been shown to impart a less invasive, dormant phenotype on metastatic cancer cells. We investigated whether hepatic nonparenchymal cells (NPCs) contributed to metastatic breast cancer cell outgrowth and a mesenchymal phenotypic shift indicative of emergence. Co-culture experiments of primary human hepatocytes, NPCs or endothelial cell lines (TMNK-1 or HMEC-1) and breast cancer cell lines (MCF7 or MDA-MB-231) were conducted. Exposure of carcinoma cells to NPC-conditioned medium isolated soluble factors contributing to outgrowth.

- We conclude that NPCs in the metastatic hepatic niche secrete factors that can induce a partial mesenchymal shift in epithelial breast cancer cells thus initiating outgrowth, and that this is in part mediated by EGFR activation. These data suggest that changes in the parenchymal cell and NPC ratios (or activation status) in the liver metastatic microenvironment may contribute to emergence from metastatic dormancy“ [15]. 
- Osada S et al.: "Colorectal cancer (CRC) is one of the most common cancers worldwide, and a strategy to assess and control liver metastasis will be critical to control patient prognosis. To evaluate therapeutic approaches, the biological responses associated with hepatectomy were studied with a focus on hepatocyte growth factor (HGF). In cases where tumors recurred within 6 months (early recurrence cases), the c-Met value in tumor tissue was higher than in cases with no recurrence, even in cases where there was no tendency for an increasing rate of HGF.The present study demonstrated that cancer with high c-Met expression and under high level of its ligand, HGF, led to recurrence soon after hepatectomy, leading to unfavorable patient prognosis. If pathological or biochemical factors from resected specimens could help identify patients with a high risk of relapse, innovative adjuvant chemotherapy protocols could be initiated" [16].

- According Filipa Macedo et al.: "Bone metastasis are almost always multiple and involve axial skeleton.4 It has been suggested that this distribution might be in relation to the hematopoietically active red bone marrow. There exists a paravertebral network that may play a role in the development of bone metastasis. This theory is supported by the high incidence of bone metastases without corresponding lesions in the lung (suggest an alternative pathway of spread). In addiction, the microenvironment must be favorable for tumor cell survival. Once the tumor cell is in circulation, it needs: Vascular adhesion and extravasation: the cell interacts with endothelium in order to extravasate and stay in a specific tissue. Chemoattractive and adhesion molecules play a fundamental role in this selective retention of cancer cells in bone marrow vasculature. Cancer cells use equivalent molecules to vascular cell adhesion molecules (VCAM) and E-selectin to adhere to endothelium. We also know that chemokines, integrins, osteopontin, bone sialoprotein and type I collagen are critical for organ colonization by cancer cells. Examples of such interactions are: expression of CXCR4 by neuroblastoma tumors that mediates the attachment to stromal-cell derived factor 1 in bone (SDF1 or CXCL12); expression of RANK by BC that mediates the attachment to RANKL in bone; expression of sialoprotein by non-small cell lung cancer that facilitate binding to collagen type I in bone. Micro-environmental support: The seed-and-soil hypothesis tells us that the microenvironment provides a fertile ground (the soil), for the survival and growth of metastatic cancer cells (the seed). The bone formation and reabsorption release and activate survival and growth promoting factors that may contribute to bone metastases development. Epithelial - Mesenchymal transition: Normal cell can lose their epithelial features and acquire mesenchymal characteristics. This process is called Epithelial-Mesenchymal Transition, and enables epithelial cells to migrate to a new environment. This occurs mainly during embryogenesis, but in cancer cells this process confers the invasive phenotype" [17].

- $\quad$ T.N. Seyfried et al.: "to complete the metastatic cascade, cancer cells must detach from the primary tumor, intravasate into the circulatory/lymphatic systems, evade immune attack, extravasate at distant capillary beds, and invade and proliferate in distant organs. Several hypotheses to explain the origin of cancer metastasis: an epithelial mesenchymal transition, an accumulation of mutations in stem cells, a macrophage facilitation process, and a macrophage origin involving either transformation or fusion hybridization with neoplastic cells. Many properties of metastatic cancer cells are also seen in normal macrophages. A macrophage origin of metastasis can also explain the long-standing "seed and soil" hypothesis and the absence of metastasis in plant cancers. Metastasis is responsible for about $90 \%$ of cancer deaths. Metastatic cells also establish a microenvironment that facilitates angiogenesis and proliferation, resulting in secondary tumors.

Tumor cells that are naturally metastatic should not require intravenous injection to initiate the metastatic phenotype. ttumor cells spread naturally from the primary tumor site to secondary. Migration of cells into scratches or in Boyden chambers might or might not be related to the phenomenon in vivo. Origin of Metastatic Cancer Cells 
Epithelial to Mesenchymal Transition (EMT): The EMT posits that metastatic cells arise from either epithelial stem cells or differentiated epithelial cells through a stepwise accumulation of gene mutations that eventually transform the epithelial cell into a tumor cell with mesenchymal features.

Many cancers arise in epithelial tissues where abnormalities in cell-cell and cellmatrix interactions occur during tumor progression Neoplastic cells emerge that appear as mesenchymal cells which lack cell-cell adhesion, are dysmorphic in shape, and spread to distant organs. According to J. P.Thiery, normal epithelia lined by a basement membrane can proliferate locally to give rise to an adenoma. Further transformation by epigenetic changes and genetic alterations leads to a carcinoma in situ, still outlined by an intact basement membrane. Local dissemination of carcinoma cells, possibly through an EMT, as the basement membrane becomes fragmented. The invasive carcinoma cells (red) then intravasate into lymph or blood vessels, allowing their passive transport to distant organs. At secondary sites, solitary carcinoma cells extravasate, remain solitary (micrometastasis), or form a new carcinoma through an MET.

The idea for the EMT arose from attempts to draw parallels between the behavior of normal cells during metazoan morphogenesis and the behavior of cancer cells during tumor progression. Adaptation of the EMT into the gene theory of cancer suggested that metastasis is the endpoint of a series of genomic alterations and clonal selection. This then provided the neoplastic cells with a growth advantage over normal cells. It is difficult to understand how a collection of gene mutations, many are random and deleterious, could produce cells with the capacity to detach from the primary tumor, intravasate into the circulation and lymphatic systems, evade immune attack, extravasate at distant capillary beds, and recapitulate epithelial characteristics following invasion and proliferation in distant organs. The recapitulation of epithelial characteristics at distant secondary sites is referred to as the mesenchymal epithelial transition (MET) and is thought to involve a reversal of the changes responsible for the EMT.

In the VM mouse model of systemic metastasis suggest that random mutations and EMT are not required for the origin of metastasis. Many of the gene expression profiles observed in metastatic cancers are similar to those associated with the function of macrophages or other fusogenic cells of the immune system. Evidence suggests that cancer is not genetic but a metabolic disease involving respiratory insufficiency with compensatory fermentation. The genomic instability seen in tumor cells arises as a downstream epiphenomenon of the underlying metabolic defects.

Several researcher hold that metastatic cancer cells arise from populations of tissue stem cells. Most tissues contain cells in semi-differentiated states that can replace dead or damaged cells due to natural wear and tear. These undifferentiated or semi-differentiated cells are often referred to as tissue stem cells and are considered by many to be the origin of metastatic cancers. Similarities in gene expression and biological characteristics are often seen in stem cells and cancer cells. Tumor cells express characteristics of undifferentiated stem cells come from the fact that embryonic stem cells and tumor cells can use anaerobic energy (fermentation) for metabolism. Telomerase activity, which is higher in tumor cells than in normal cells, is linked to fermentation energy. It is therefore not surprising that numerous genetic and biochemical phenotypes are shared between tumor cells and stem cells, as most tumor cells also use energy from fermentation for their survival and growth.

As stem cells are known for their ability to proliferate and migrate during tissue morphogenesis and differentiation, it was reasonable that genetic damage to stem cells could give rise to metastatic cancers in various tissues (Many tumor cells with stem cell properties do not express systemic metastasis). Many of the human xenograft tumor models rarely show systemic metastasis when grown in the immune compromised mouse host despite expressing several of the Hanahan and Weinberg cancer hallmarks. 
While metastatic cancers can express properties of stem cells, expression of stem cell properties is not synonymous with expression of metastasis.

Tumors derived from hematopoietic stem cells, may be an exception Hematopoietic stem cells can give rise to myeloid cells, which we consider the origin of most metastatic cancers. Only mouse tumor cells expressing characteristics of macrophages showed systemic metastasis. Macrophage Facilitation of Metastasis: many malignant tumors contain significant $n$. of macrophages and other cells of the stroma. The macrophages present in tumors are generally referred to as tumor-associated macrophages (TAM). That can establish the pre-metastatic niche, while enhancing tumor inflammation and angiogenesis and facilitate the metastatic cascade.

In this model while gene mutations are still thought to initiate neoplasia, stromal macrophages acting as cellular chaperones that facilitate tumor development, progression, and metastasis.

The stromal TAM are viewed as essential participants in all phases of metastasis, but are not considered neoplastic themselves. Many human metastatic tumors also contain neoplastic cells with macrophage properties. It is not easy to distinguish neoplastic from non-neoplastic macrophages in the inflamed tumor microenvironment (similar in gene expression, morphology, and function). We consider metastatic cells themselves as derived from macrophages or other similar cells of myeloid origin. TAM secrete a proteases to breakdown the basement membrane around areas of proliferating tumor cells (e.g., ductal carcinoma in situ in the breast), thereby prompting their escape into the surrounding stroma where they show deregulated growth, angiogenesis: In areas of transient (avascular) and chronic (perinecrotic) tumor hypoxia, macrophages cooperate with tumor cells to induce a vascular supply for the area by up-regulating a number of angiogenic growth factors and enzymes. These diffuse away from the hypoxic area and, together with other pro-angiogenic stimuli in the tumor microenvironment, stimulate endothelial cells in neighboring, vascularized areas to migrate, proliferate, and differentiate into new vessels., immunosuppression: Macrophages in hypoxic areas secrete factors that suppress the antitumor functions of immune effectors within the tumor. , metastasis: A subpopulation of TAM associated with tumor vessels secretes factors like epidermal growth factor (EGF) to guide tumor cells in the stroma toward blood vessels where they then escape into the circulation.

TAM secrete growth factors in the stromal compartment to stimulate tumor cell division and/or undefined factors that promote tumor cell motility. Myeloid Cell Origin of Metastasis: According to our hypothesis, metastatic cancers arise from respiratory insufficiency in myeloid cells or in their lineage descendants, e.g., macrophages, dendritic cells, or lymphocytes. Chronic inflammation can damage mitochondrial respiration in activated macrophage. Many metastatic cancers express aerobic glycolysis (Warburg effect), which can be detected in PET scans. Aerobic glycolysis in tumor cells arises ultimately from insufficient respiration. Fusion hybridization between macrophages and non-metastatic cancer stem cells also blurs the boundaries between the nuclear and cytoplasmic contribution to the metastatic phenotype. Other hypothesis to the view that normal macrophages facilitate the metastatic spread of neoplastic stem cells; the myeloid hypothesis of metastasis suggests that metastatic cancer cells arise directly from cells of myeloid origin or from hybrid cells following fusion between macrophages and non-metastatic stem cells.

The myeloid cell origin of metastasis would also encompass the macrophage fusion hypothesis of metastatic cancer, since it is the properties of macrophages that contribute to the metastatic cascade. Myeloid cells are already mesenchymal cells and would not, therefore, require the complicated genetic mechanisms proposed for the EMT in order to metastasize. Macrophages arise from the myeloid lineage and have long been considered the origin of human metastatic cancer. Macrophages can fuse 
with epithelial cells within the inflamed microenvironment thus manifesting properties of both the epithelial cell and the macrophage in the fusion hybrids.

Tarin states: "it would appear that tumour metastasis first appears in the lower chordates in parallel with the origin of lymphocytes and this may indicate that metastasis cannot occur until an organism has evolved the genes for lymphocyte trafficking." Macrophages present ability to migrate, to change shape, and to secrete growth factors and cytokines (behaviors are also the recognized behaviors of metastatic cells). Macrophages manifest two distinct polarization phenotypes: the classically activated (M1 phenotype) and the alternatively activated (M2 phenotype). Macrophages acquire the M1 phenotype in response to pro-inflammatory molecules and release inflammatory cytokines, reactive oxygen species, and nitric oxid In contrast, macrophages acquire the M2 phenotype in response to anti-inflammatory molecules such as IL-4, IL 13, IL-10 and to apoptotic cells. M2 macrophages promote tissue remodeling and repair, but are immunosuppressive and poor antigen presenters. The M1 and the M2 macrophages play distinct roles during tumor initiation and malignant progression, macrophage-epithelial cell fusions can involve either activation state.

M1 macrophages facilitate the early stages of tumorigenesis through the creation of an inflammatory microenvironment that can produce nuclear and mitochondrial damage. TAM can also undergo a phenotypic switch to the M2 phenotype during tumor progression. The TAM population comprising M2 macrophages scavenge cellular debris, promote tumor growth, and enhance angiogenesis. M2 macrophages also fuse with tumor cells, thus, expressing characteristics of both cell types. Many of the myeloid/macrophage cells seen within human tumors are also part of the malignant cell population. Aichel first proposed over a century ago that tumor progression involved fusion between leukocytes and somatic cells. Several human metastatic cancers express multiple molecular and behavioral characteristics of macrophages (phagocytosis, cell-cell fusion, antigen expression).

Tarin: the expression of osteopontin and CD44 as important for the regulatory gene group/network associated with metastasis. Macrophages express most hallmarks of metastatic tumor cells when responding to tissue injury or disease. Monocytes (derived from hematopoietic bone marrow cells) extravasate from the vasculature and are recruited to the wound via cytokines released from the damaged tissue.

Within the wound, monocytes differentiate into alternatively-activated macrophages and dendritic cells where they release a variety of pro-angiogenic molecules including vascular endothelial growth factor, fibroblast growth factor, and platelet derived GF. M2 macrophages also actively phagocytize dead cells and cellular debris. Macrophages intravasate back into the circulation where they travel to the lymph nodes to participate in the immune response. Some macrophages also migrate to lymph nodes and differentiate into dendritic cells. Normal macrophages are capable of expressing all hallmarks of metastatic cancer cells including tissue invasion, release of proangiogenic molecules/cytokines, survival in hypoxic and necrotic environments, intravasation into the circulatory/lymphatic systems, and extravasation from these systems at distant locations. An EMT is not necessary to explain these behaviors, as they are already the evolutionary programmed behaviors of macrophages.

Phagocytosis involves the engulfment and ingestion of extracellular material, and is a specialized behavior of M2 macrophages and other professional phagocytes. This process is essential for maintaining tissue homeostasis by clearing apoptotic cells, cellular debris, and invading pathogens. Like M2 macrophages, many malignant tumor cells are phagocytic both in vitro and in vivo. This cellular phenotype resulted from the ingested material pushing the nucleus to the periphery of the phagocytic cell. These cells were commonly referred to as either "birds-eye" or "signet-ring" cells. Phagocytic/cannibalistic phenomenon is commonly seen in feeding microorganisms 
and in malignant human tumor cells. T-cells are thought to target and kill tumor cells. Some tumor cells can eat natural killer cells. Melanocytes are the resident macrophages of the skin. Expression of cathepsins B and D are elevated in the phagocytic melanoma cells just as they are in malignant melanomas. Reports have described the phagocytic behaviors seen in aggressive human cancers. We identified two spontaneous invasive/ metastatic murine brain tumors (VM-M2 and VM-M3) that express many macrophage characteristics including phagocytosis. These metastatic tumor cells engulf fluorescent beads. Interesting features of these natural mouse brain tumors was their metastatic behavior when grown outside the central nervous system. The cells spread to multiple organ systems following implantation into most extracranial sites.

While extracranial metastasis of CNS tumors is not common, many gliomas, especially glioblastoma multiforme (GBM), are highly metastatic if the tumor cells gain access to extraneural tissue. Investigators have documented the metastatic behavior of malignant brain cancers, especially GBM.The VM-M2 and VM-M3 tumors replicate this feature of GBM behavior. Recipients of organs from a donor with GBM developed metastatic cancer. Neoplastic cells from this GBM metastasized from the brain and infiltrated extraneural tissues without detection. While is difficult to prove a myeloid origin of invasive GBM cells, subpopulations of neoplastic GBM cells display the phagocytic behavior of macrophages/microglia. As microglia are the resident macrophages of the brain, we considered that some of the cells in these tumors could arise from neoplastic microglia/macrophages. GBM, like many advanced metastatic human cancers, contain mixtures of numerous neoplastic cell types, many have mesenchymal properties and are of unknown cellular origin.

The original nineteenth century observations of Virchow (1863) described glioblastomas as gliosarcomas of mesenchymal origin. Many of the neoplastic mesenchymal cells seen in GBM arise from transformed macrophages or microglia that fuse with neoplastic stem cells. Phagocytic behaviors have been reported for many human cancers including skin, breast, lymphoma, lung, brain, ovarian, pancreatic, renal, endometrial, rhabdomyosarcoma, myeloma, fibrosarcoma and bladder. For most of these tumors, the phagocytic phenotype was restricted primarily to those cells that were also highly invasive and metastatic. The most potentially deadly cells within tumors are those with macrophage properties. Numerous phagocytic tumor cells were identified within metastatic breast cancer lesions and were not observed within the primary tumor of the same patient. The n. of phagocytic tumor cells present within the tumor stroma correlates with breast cancer malignancy and grade. Phagocytosis is a common macrophage phenotype seen in many metastatic human cancers.

RAW 264.7 cells are considered a normal mouse macrophage cell line and are used to study macrophage properties (that were transformed with Abelson leukemia virus and derived from BALB/c mice.) Viruses damage mitochondrial function, initial event in the transformation of a normal cell to a neoplastic. We used this cells as a control cell line for our metastatic VM-M2 and VM-M3 metastatic cancer cells. Using fluorescent microspheres, we found that the phagocytic activity of the metastatic VMM2 and VM-M3 tumor cells was similar to that of the RAW 264.7 macrophage cell line. Were there similarities between the RAW cells and the metastatic VM tumor cells for phagocytic behavior, similar in their morphology, gene expression, lipid composition? To determine whether the RAW cells were tumorigenic or non-tumorigenic, we implanted the RAW 264.7 cells subcutaneously into the flanks of immunodeficient BALB/SCID mice. The RAW cells not only formed tumors and systemic metastasis.

The RAW 264.7 macrophage cell line is highly metastatic following CNS and subcutaneous transplantation into SCID mice. The metastatic behaviors of the RAW cells also appear similar to the metastatic behaviors of the tumors. Like the VM-M2 and VM-M3 tumor cell lines, the RAW 264.7 cells express little ganglioside GM3 and 
metastasize to multiple organ systems (liver, spleen, kidney, lung, and brain) when grown subcutaneously outside the brain. Ganglioside GM3 inhibits angiogenesis and blocks tumor cell invasion. Cells with macrophage properties can give rise to metastatic cancer regardless of how the cells are classified. Fusogenicity (ability of a cell to fuse with another cell through the merging of their plasma membranes) is a Shared Behavior of Macrophages and Many Metastatic Cancer Cells.

Fusion in human cells is a highly regulated process that is essential for fertilization (sperm and egg) and skeletal muscle (myoblasts) and placenta (trophoblast) formation. During differentiation, subsets of macrophages fuse with each other to form multinucleated osteoclasts in bone or multinucleated giant cells in response to foreign bodies. Osteoclasts and giant cells have increased cell volume that facilitates engulfment of large extracellular materials. Macrophages are also thought to fuse with damaged somatic cells during the process of tissue repair. Macrophages undergo heterotypic fusion with tumor cells.

Aichel: fusion between somatic cells and leukocytes could induce aneuploidy resulting in tumors with increased malignancy.

Mekler et al: fusion of committed tumor cells with host myeloid cells would produce tumor hybrids capable of migrating throughout the body and invading distant organs. Inflammation, and radiation increases the fusion hybrid process.

Pawelek ET: fusion hybrids could account for the diversity of cell phenotypes observed within tumors. Fusion between neoplastic tumor cells and myeloid cells, with subsequent nuclear fusion, could produce new phenotypes in the absence of new mutations, as the hybrids would express genetic and functional traits of both parental cells. These neoplastic hybrids would express the macrophages characteristics to intravasate, extravasate, and migrate to distant organs while also possessing the unlimited proliferative potential of the cancer cells. Since myeloid cells are part of the immune system, it would be easy to see how tumor hybrids would also be able to evade immune surveillance. Many tumor cells are fusogenic. These cells are found in a wide-variety of cancer types including, melanoma, breast, renal, liver, gall bladder, lymphoma and brain. Human glioma cells, when implanted within the cheeks of hamsters, spontaneously fused with non-tumorigenic host cells, resulting in metastatic hybrid humanhamster tumor cells. CXCR4 is expressed in our highly metastatic VM-M2 macrophage/microglial tumor cells. Reports for fusogenic cancers described fusions between lymphomas and myeloid cells.Spontaneous in vivo fusion between the non-metastatic murine MDW4 lymphoma and host bone marrow cells resulted in aneuploid metastatic tumor cells. Seyfried: the horizontal transfer of information from one cell to another during tumor progression was an example of Lamarckian inheritance and that the evolutionary concepts of Lamarck could better explain tumor progression than Darwin.

Munzarova et al.: numerous traits expressed in macrophages were expressed in metastatic melanoma cells: the tumor metastasis could result from fusions between tumor cells and macrophages.

Pawelek etal: showed that the majority of macrophage-melanoma hybrids displayed increased metastatic potential when grown in vivo. Tumor-associated macrophages promote tumor progression through the release of cytokines, and pro-angiogenic and pro-metastatic molecules. The fusion of cancer cells with tissue macrophages could also accelerate tumor progression. Fusion among tumor cells in human solid tumors is difficult to detect. Several reports provide evidence for fusions between tumor cells and myeloid cells in human bone marrow transplant (BMT) recipients. Such fusions would accelerate tumor progression. Wong et other conducted parabiosis experiments, where one mouse is surgically attached to another mouse, to show how bone marrowderived cells of one mouse fuse with intestinal tumor cells of the other mouse. 
They identified the macrophage as the driver for this process and showed that the fused hybrid cells retained a transcriptome identity characteristic of both parental derivatives, while also expressing unique transcripts. Fusions between macrophages and tumor cells, within the inflamed wound environment, could give rise to the metastatic phenotype of cancer cells thus enhancing malignant progression. Radiation therapy and immuno-suppression can increase the incidence of metastatic cancers. DNA analysis of micro-dissected metastatic cells from a child diagnosed with renal cell carcinoma after a bone marrow transplant revealed DNA from both the BMT donor and the recipient in the metastatic cells. Bone M. and tumor cell hybrids were also identified in a female who developed renal carcinoma after receiving a BMT from a male donor. (Genetic evidence that spontaneous fusions can occur between human myeloid cells and tumor cells). Multinucleated giant cells, a signature of hybrid formation, are frequently seen in human cancers suggesting that cell fusions are not rare.

Myeloid Biomarkers Expressed in Tumor Cells: TAMs are often correlated with a poor patient prognosis, tumor biopsies are frequently evaluated for macrophage markers. TAM are generally thought to comprise the macrophage antigen-expressing cells observed within the tumor stroma, reports show that macrophage-specific antigens and biomarkers are expressed on a wide variety of human cancer cells. Ruff: macrophage antigens (CD26, C3bi and CD11b) were expressed on the tumor cells from small cell lung carcinoma (SCLC). It is important to note that the macrophage antigens were also expressed in the cultured tumor cells themselves. This tumor cell expression was confirmed from in vivo tissue preparations.

This eliminated the possibility that the antigen expression was derived from TAM. These investigators concluded that the SCLC tumor cells in their specimens were not of lung epithelial origin, but rather were of "myeloid origin". The myeloid properties of the SCLC were derived from fusions of macrophages and neoplastic lung epithelial cells. SCLC, myeloid-associated antigens (CD14 and CD11b) were also expressed in five metastatic breast cancer cell lines. None of the breast cancer cell lines, however, expressed markers for B-cells or T-cells. Evidence for a mesenchymal origin of metastatic cancer comes from tissue microarray analysis of 127 breast cancer patients. Pathology confirmed that the staining was localized to the tumor cells and not solely to the tumor infiltrating macrophages. Cancers that contain CD163-expressing tumor cells have a more advanced histological grade, enhanced metastasis, and reduced patient survival. Tumor cells expressing macrophage antigens could be identified in more than half of breast cancer patients.

As in breast cancer patients, CD163 was expressed on tumor cells in many patients with bladder and rectal cancer. CD163 expression was found in 31\% of the rectal tumors from patients in the preoperative irradiation group, but was expressed in only $17 \%$ in the non-irradiation group. Prognosis was also worse for those patients with CD163 + cancer cells than in those patients with CD163-negative cancer cells. Inflammation and radiation is known to enhance formation of macrophage epithelial cell fusion hybrids.

Maniecki et al.: expression of CD163 could be a common phenotype of many metastatic cancers arising from heterotypic cell fusions between tumor cells and macrophages. The findings in these metastatic cancers are consistent with the origin of metastatic cells from transformed macrophages or from macrophage fusion hybrid cells, which are increased from radiation and inflammation. Radiation therapy can help some cancer patients, radiation therapy will also enhance mitochondrial damage and fusion hybridization thus potentially making the disease much worse. The role of radiation in inducing tumor cell-macrophage fusions and in exacerbating the metastatic properties of some cancers. Macrophage antigens, which are associated with enhanced metastasis and poor prognosis, are expressed on the tumor cells of patients with breast, bladder, rectal cancers, and brain cancers. Cathepsins, Macrophages express 
high levels of lysosomal-enriched cathepsins, which facilitate the digestion of proteins ingested following phagocytosis or pinocytosis. lysosomal cathepsins D and B are prognostic factors in cancer patients. A high content of these enzymes in tumors of the head and neck, breast, brain, colon, or endometrium was considered a sign for high malignancy, high metastasis, and overall poor prognosis.Cathepsins, activated macrophages also express ezrin.

The ezrin-radixin-moesin is a family of molecules that play essential roles in tissue remodeling by linking the cell surface with the actin cytoskeleton and facilitating signaltransduction pathways..The transition from epithelial to mesenchymal phenotype is associated with downregulation of the cell adhesion molecule, E-cadherin. Iron deficient anemia is a co-morbid trait in many patients with metastatic cancers. Hepcidin is a regulator of iron metabolism and plasma iron levels by controlling the efflux of iron from enterocytes, hepatocytes, and macrophages and by internalizing and degrading the iron exporter, ferroportin. Hepcidin might contribute to the systemic anemia in colorectal cancer patients by acting at the level of the macrophage. Activated macrophages express IL-6, which induces expression of hepcidin. Macrophages are the major cell type responsible for systemic iron recycling. Ward et al: cancer is a disease of myeloid cells especially macrophages. Many characteristics of metastatic cancers can be explained once it becomes recognized that metastatic cancer is a macrophage metabolic disease. Iron deficient anemia should not be unexpected for metastatic cancers derived from transformed macrophages or macrophage fusion hybrids.

Carcinoma of unknown primary (CUP) is a systemic metastatic disease without an identifiable primary tumor and is often associated with poor prognosis. About $5 \%$ of all newly diagnosed cancers are classified as CUP. These cancers are classified as adenocarcinomas, squamous cell carcinomas, poorly differentiated carcinoma, and neuroendocrine carcinomas. Metastasize before the primary tumor has had time to develop into a macroscopic lesion. Due to high aggressiveness, some CUPs could arise from macrophage fusion hybrids. Many Metastatic Cancers Express Multiple Macrophage Properties (express multiple myeloid characteristics). Many phagocytic / fusogenic tumors also express myeloid antigens.

Pawelek et al.: Cell fusion events involving macrophages can give rise to cells that metastasize, indicates that nearly all cancers are a type of mitochondrial disease arising from respiratory insufficiency. This damage leads to fermentation as a compensatory source of energy (Warburg THEORY). When permanent respiratory damage occurs in cells of myeloid origin including hematopoietic stem cells and their fusion hybrids, metastasis would be a potential outcome. Mitochondria from a broad range of metastatic cancers are abnormal and incapable of generating energy through normal respiration. Energy through fermentation is the single most common hallmark of all cancer cells including those with metastatic potential. This phenotype arises from mitochondrial dysfunction. Mitochondrial damage can arise in any cell within the inflammatory microenvironment of the incipient tumor including TAM, homotypic fusion hybrids of hematopoietic cells, or heterotypic fusion hybrids of macrophages and neoplastic epithelial cells. The end result would be cells with metastatic potential.

Metastatic cells will differ in their morphology from one organ system to the next, they all suffer from the common malady of insufficient respiration. The tumor microenvironment consists of numerous mitochondria damaging elements, which could impair mitochondria energy production in TAM and tissue macrophages. This would produce genetic instability through the mitochondrial stress or retrograde signaling (RTG) response. Fusions between macrophages or between macrophages and cancer stem cells could result in cells expressing both the tumor and macrophage genomes. The end result would be cells that can survive in hypoxic environments, can proliferate, and can spread to multiple sites through the circulation. 
Respiratory Damage in Macrophage Fusion Hybrids. Normal mitochondrial function suppresses tumorigenesis. Nuclear gene mutations alone cannot account for the origin or progression of cancer. Normal respiration would initially suppress tumorigenicity in fused hybrids, persistent or recurrent inflammation in the microenvironment will eventually damage the majority of mitochondria in the fused hybrids, initiating metastasis. As macrophages evolved to survive in hypoxic and inflammatory environments, considerable time and iterative damage to respiration would be necessary to initiate tumorigenesis in the fusion hybrids. Radiation exposure would not only enhance fusion hybrid formation, but would also damage respiration thus leading to compensatory fermentation and the onset of tumorigenesis. As respiration is responsible for maintaining genomic stability and the differentiated state, respiratory insufficiency will eventually induce the default state of unbridled proliferation and genomic instability. If this occurs in cells of myeloid origin like macrophages, then emergence of cells with enhanced metastatic potential would be a predicted outcome. Macrophages are genetically programmed to exist in the circulation and to enter and exit tissues. The dysregulated behavior of these cells through corrupted energy metabolism would have dire consequences. Oncogene activation and tumor suppressor-gene inactivation are required to maintain energy production through fermentation following irreversible injury to oxidative phosphorylation Enhanced glucose uptake seen in metastatic lesions under PET scanning is indicative of enhanced glycolysis and abnormal energy metabolism.

Metastatic tumor cells do not invade distant organs randomly. Metastatic cancer cells invade in a non-random pattern with lung, liver, and bone as primary sites of metastases. Surgeon, S. Paget, was the first to record this phenomenon in his "seed and soil" hypothesis of breast cancer metastasis. He proposed that certain tumor cells (the seed) have a preferential affinity to invade certain organs (the soil). The non-random dissemination of metastatic cancer cells has engaged the attention of numerous investigators for decades, no credible genetic mechanism has been able to account for the phenomenon. The seed and soil hypothesis is extremely difficult to explain if cancer is viewed as a genetic disease. There are no clear connections between the non-random invasion of distant organs and the genetic abnormalities in metastatic cells. Respiratory insufficiency in cells of myeloid origin can explain the seed and soil THEORY. Mature cells of monocyte origin like macrophage enter and engraft tissues in a non-random manner. Some macrophage populations in liver are regularly replaced with bone marrow derived monocytic cells, whereas other macrophage populations are more permanent and require fewer turnovers.

Many metastatic cells express characteristics of macrophages. Macrophage turnover should be greater in tissues like liver and lung where the degree of bacterial exposure and the wear-and-tare on the resident macrophage populations is considerable. This could explain why these organs are a preferred soil of many metastatic cancer cells. Bone marrow should also be a common target of metastatic cells because this site is the origin of the hematopoietic stem cells, which give rise to myeloid cells. Liver, lung, and bone are also preferential sites for metastatic spread for the VM mouse tumor cells.

The natural tumors in the VM mouse, which preferentially home to these tissues, are an excellent model for metastatic cancer. Because the metastatic cells express insufficient respiration with compensatory fermentation, these cells will enter their default state of proliferation, as would any neoplastic cell. Organs receiving high macrophage turnover, macrophages also target sites of inflammation and injury.

Metastatic cancer cells from lung and breast can appear in the mouth following recent tooth extraction or along needle tracts following biopsy. An unhealed wound is an ideal "soil" for macrophage infiltration. (As inflammatory "oncotaxis" and can explain in part the seed and soil hypothesis). If metastasis were a metabolic disease of myeloid cells, then the appearance of metastatic cells in recent tooth extraction or 
wounds would not be unexpected. The non-random pattern of metastasis to visceral organs, bone marrow, and wounds is consistent with a macrophage origin of metastasis. In contrast to the EMT, the MET involves proliferation and re-expression of epithelial characteristics following extravasation, invasion, and proliferation at distant cites. The MET is a reversibility of the EMT.

A myeloid cell origin of metastasis provides a credible explanation of metastasis than the MET/MET. Metastatic cells arising from myeloid cell fusions would retain the genetic architecture necessary for entering and exiting the circulation at recognized sites. It is not necessary to construct complicated mutation based regulatory systems to explain this. Macrophages naturally enter and exit the circulatory and lymphatic systems. The circulatory system is not a "hostile" environment for cells in the macrophage lineage, as macrophage precursors, i.e., monocytes, exist naturally in the circulation. These cells also express the cell-surface adhesion molecules (selectins) necessary for extravasation at designated organs. They express the batteries of metalloproteases necessary for degradation of basement membranes and invasion. When these capabilities occur together with impaired respiration, dysregulated proliferation would be an expected outcome. While these properties certainly implicate myeloid cells as the origin of metastatic cells, the fusogenic properties of myeloid cells can also explain how metastatic cells can recapitulate the epithelial characteristics of the primary tumor at secondary sites.

Fusion hybrid hypothesis of cancer cell metastasis. Metastatic cancer cells arise following direct transformation or following fusion hybridization between neoplastic epithelial cells and myeloid cells (macrophages). Macrophages are known to invade in situ carcinoma as if it were an unhealed wound. This creates a protracted inflammatory microenvironment leading to fusion hybridization between the neoplastic epithelial cell and the macrophage. Inflammation damages mitochondria leading to enhanced fermentation and acidification of microenvironment. Mitochondrial damage leading to respiratory insufficiency as driver for the neoplastic transformation of the epithelial cell and of the fusion hybrids As macrophages are already mesenchymal cells that naturally possess the capability to enter (intravasate) and exit (extravasate) the circulation, the neoplastic fusion hybrid will behave as a rogue macrophage.

The fusogenic properties of macrophage cells can explain how metastatic cells can recapitulate the epithelial characteristics of the primary tumor at secondary micrometastatic growth sites. Studies of fusion hybrids showed that functional hepatocytes could be derived from bone marrow derived macrophages or myelomonocytic cells following cell fusions. Rizvi, et al.: the expression of epithelial characteristics were found in fusion hybrids between bone marrow-derived cells and either normal epithelium or neoplastic intestinal epithelium. Wong and colleagues showed how macrophage/epithelial cell hybrids could recapitulate phenotypes of epithelial cells while retaining the properties of macrophages.

Phenotypes of epithelial cells and macrophages can be maintained in fusion hybrids of macrophages and intestinal epithelial tumor cells. Fusions of activated macrophages with epithelial cells in the primary tumor microenvironment will bestow the capability of the fused cells to degrade basement membranes, to enter and exit the circulatory and lymphatic systems, and to recapitulate the epithelial characteristics of the primary tumor at distant secondary sites. The dysregulated growth at secondary sites is the consequence of damaged respiration in these cells. The origin of metastatic cells from macrophage fusion hybrids with dysfunctional mitochondria can explain the metastasis.

Genetic heterogeneity is observed in comparing tumor tissue from primary growth sites with tissue from distant metastases. (Is seen not only between patients with similar tumor histopathology. Also for the tumors growing at different sites within the 
same patient). Every type of genetic heterogeneity imaginable from point mutations to major genomic rearrangements can be found in metastatic and highly invasive cancers including those from breast, brain, and pancreas.If the spread of metastatic cells to some organs (liver and lung) occurs earlier than spread to other organs, it is possible that genetic heterogeneity would be greater in these organs than in organs that receive metastatic cells later in the disease progression. This is expected if the number of divisions is greater for tumor cells that arrive earlier in these organs than for tumor cells that arrive later in other organs.

Campbell et al.: "the biological pathways underlying these forms of genomic instability remain unclear." As genomic stability is dependent on normal mitochondrial function, it should not be surprising that there is a "richness of genetic variation in cancer" as Campbell and co-workers describe.

The gene mutations also arise as downstream epiphenomena of respiratory insufficiency with compensatory fermentation. The crown-gall disease in plants shares many features with tumors in animals. Crown-gall tumors arise form bacterial infections that enter damaged areas of the plant leading to plant cell proliferation. Robinson first suggested that Warburg's cancer theory might account for the abnormal cell proliferation in crown-gall tumors following bacterial damage to respiration in the affected plant cells. Defects in mitochondrial morphology and energy metabolism were later described in crown-gall tumors. The crown-gall tumors express four of the Hanahan and Weinberg hallmarks of cancer, i.e., self-sufficiency in growth signals, insensitivity to growth inhibitory (anti-growth) signals, evasion of programmed cell death (apoptosis), and limitless replicative potential.

(These do not express invasion or metastasis. With the exception of metastasis, the abnormalities in growth are similar in crown-gall disease and in animal tumors). If metastasis arises from damaged respiration in macrophages or in their fusion hybrids, then it becomes clear why the grown-gall tumors do not display invasion or metastasis despite expressing other hallmarks of tumors. The crown-gall tumors do not metastasize because they do not have macrophages or myeloid cells as part of their immune system Tarin's hypothesis, "that metastasis cannot occur until an organism has evolved the genes for lymphocyte trafficking". Plants have not evolved these genes as far as we know. Metastasis occurs predominantly in cells that express properties of macrophages.

As a metabolic disease, most if not all cancers can be managed by targeting those fuels necessary for their proliferation and survival. The goal is to first transition energy metabolism of all normal cells of the body to ketone bodies, which tumor cells cannot effectively use for energy. Most tumor cells require glucose for energy through glycolysis. Glutamine is a major metabolic fuel for many cells of the immune system. Simultaneous targeting of glucose and glutamine under calorie restriction could significantly reduce systemic metastatic cancer in the VM-M2 mouse model. Targeting these fuels was more effective in blocking metastasis than was using the well-known toxic chemotherapies. A transition from an epithelial cell to a mesenchymal cell is considered characteristic of metastasis. It is improbable that random mutations acquired through a Darwinian selection process could account for all of the myeloid-cell behaviors necessary for the completion of the metastatic cascade. As an alternative to a series of gain-offunction mutations and clonal selection, we propose that the metastatic mesenchymal phenotype arises initially from respiratory damage in macrophages or in epithelialmacrophage fusion hybrids, followed by compensatory fermentation. This would produce the metastatic lesion images seen on PET. Inflammation and radiation damage enhances hybridization while also damaging mitochondrial function over time" [18].

- Jiang WG et al.: "Hepatocyte growth factor (HGF) is the most potent stimulator of hepatocyte growth and DNA synthesis identified; it is now known to be the same 
molecule as scatter factor, which increases the motility of a variety of cell types. HGF is becoming recognized as one of the most important factors in the regulation of liver regeneration after surgical resection or chemical damage. HGF is produced by several tissues, including neoplasms; it can therefore provide a stimulus for increased motility of malignant cells by both a paracrine and autocrine mechanism. The receptor for HGF has been identified as the product of the oncogene c-met, raising the possibility that this gene plays a key role in facilitating cellular invasion. HGF may therefore be important not only for liver cell growth but also in metastasis. This article summarizes the research on HGF, and presents evidence that strongly implicates this factor in liver regeneration and cancer invasion and metastasis" [19].

- Amanda M Clark et al.: "The liver is a highly metastasis-permissive organ, tumor seeding of which usually portends mortality. Its unique and diverse architectural and cellular composition enable the liver to undertake numerous specialized functions, however, this distinctive biology, notably its hemodynamic features and unique microenvironment, renders the liver intrinsically hospitable to disseminated tumor cells.

- Bidirectional interactions between the disseminated tumor cells and the unique resident cell populations of the liver; notably, parenchymal hepatocytes and non-parenchymal liver sinusoidal endothelial, Kupffer, and hepatic stellate cells. Understanding the early steps in the metastatic seeding, including the decision to undergo dormancy versus outgrowth, has been difficult to study in 2D culture systems and animals due to numerous limitations. In response, tissue-engineered biomimetic systems have emerged. At the cutting-edge of these developments are ex vivo 'microphysiological systems' (MPS) which are cellular constructs designed to faithfully recapitulate the structure and function of a human organ or organ regions on a milli- to micro-scale level and can be made all human to maintain species-specific interactions. Hepatic MPSs are particularly attractive for studying metastases as in addition to the liver being a main site of metastatic seeding, it is also the principal site of drug metabolism and therapy-limiting toxicities. Thus, using these hepatic MPSs will enable not only an enhanced understanding of the fundamental aspects of metastasis also for therapeutic agents to be studied" [20].

- Takeda A et al.: "Cancer metastasis is a highly complex process that involves aberrations in gene expression by cancer cells leading to transformation, growth, angiogenesis, invasion, and dissemination, survival in the circulation, and subsequent attachment and growth in the organ of metastasis. Angiogenesis facilitates metastasis formation by providing a mechanism to (1) increase the likelihood of tumor cells entering the blood circulation and (2) provide nutrients and oxygen for growth at the metastatic site. The formation and establishment of metastatic lesions depend on the activation of multiple angiogenic pathways at both primary and metastatic sites. Vascular endothelial growth factor, interleukin-8, and platelet-derived endothelial cell growth factor are all proangiogenic factors that have been associated with liver metastasis from various primary tumor types. Iinhibition of integrins that mediate endothelial cell survival may also serve as a component of therapeutic regimens for liver metastases" [21].

- Gordon-Weeks AN et al.: "Hepatic metastases are amenable to ablation; however, many patients are not suitable candidates for such therapy and recurrence is common. The tumor microenvironment is known to be essential for metastatic growth, yet identification of plausible targets for cancer therapy in the microenvironment has proven elusive. We found that human colorectal cancer liver metastases and murine gastrointestinal experimental liver metastases are infiltrated by neutrophils. Plasticity in neutrophils has recently been shown to lead to both protumor and antitumor effects. Here, neutrophils promoted the growth of hepatic metastases, 
given that depletion of neutrophils in already established, experimental, murine liver metastases led to diminished metastatic growth. Decreased growth was associated with reductions in vascular density and branching suggestive of vessel normalization. Metastasis-associated neutrophils expressed substantially more fibroblast growth factor 2 (FGF2) than naïve neutrophils, indicating neutrophil polarization by the tumor microenvironment. Administration of FGF2 neutralizing antibody to mice bearing experimental liver metastases phenocopied neutrophil depletion by reducing liver metastatic colony growth, vascular density, and branching." Here, we show, using FGF2 as an example, that identification of factors responsible for the protumoral effects of infiltrating myeloid cells can be used to target established liver metastases. Such therapies could be utilized to limit disease progression and potentiate the effects of standard ablative therapies" [22].

- Yingjie Wu et al.: "Among the mechanisms implicated in the tumor-promoting effects of obesity, signaling by insulin-like growth factor-I (IGF-I) and insulin has received considerable attention. However, the emerging realization that obesity is associated with chronic inflammation has prompted other consideration of how the IGF-I axis may participate in cancer progression. In the present study, we used two mouse models of chronic (LID) and inducible (iLID) igf- 1 gene deficiency in the liver to investigate the role of IGF-I in regulating the host microenvironment and colorectal carcinoma growth and metastasis in obese mice. Obese mice had a heightened inflammatory response in the liver, which was abolished in mice with chronic IGF-I deficiency (LID). In control animal's changes to the hepatic microenvironment associated with obesity sustained the presence of tumor cells in the liver and increased the incidence of hepatic metastases after intrasplenic/portal inoculation of colon carcinoma cells. These changes did not occur in LID mice with chlonic IGF-1 deficiency. In contrast, these changes occured in iLID mice with acute IGF-1 deficiency, in the same manner as the control animals, revealing a fundamental difference in the nature of the requirement for IGF-1 on tumor growth and metastasis. In the setting of obesity, our findings imply that IGF- 1 is critical to activate and sustain an inflammatory response in the liver that is needed for hepatic metastasis, not only through direct, paracrine effect on tumor cell growth, but also through indirect effects involving the tumor microenvironment" [23].

- Paschos KA et al.: "Hepatic resection remains the primary potentially curative therapeutic modality for liver metastases. The regenerative process that occurs postoperatively is a complex phenomenon, orchestrated by molecular cascades involving growth factors, cytokines, proteolytic enzymes and other proteins. Unfortunately, some of these molecules, such as hepatocyte growth factor, tumour growth factor beta and matrix metalloproteinases also promote tumour growth and may contribute to the recurrence of liver metastasis. The reactivation of dormant micrometastases or the intrahepatic accumulation of circulating malignant cells has been suggested as the responsible mechanism, although not clearly understood. Current clinical and experimental research has developed inhibitors of several regenerative molecules, attempting to treat tumour reappearance within the liver. This review describes the responsible molecular pathways and the clinical importance of post-hepatectomy liver regeneration, and investigates how the regenerative process may promote metastatic tumour recurrence" [24].

- Fernandez MC et al.: "Hepatic stellate cells (HSC) play a major role in initiating the liver fibrogenic (wounding) response of the liver and can also orchestrate a prometastatic microenvironment in the liver in response to invading cancer cells. Here we explored the role of the hepatic stellate cells in colon carcinoma liver metastasis with emphasis on the contribution of the insulin-like growth factor (IGF) axis to their activation and function. To this end, we used mice with a Tamoxifen inducible liver IGF-I deficiency. We found that in mice with a sustained IGF-I deficiency, recruitment and 
activation of HSC into tumor-infiltrated areas of the liver were markedly diminished, resulting in decreased collagen deposition and reduced tumor expansion. In addition, IGF-I could rescue HSC from apoptosis induced by pro-inflammatory factors such as TNF- $\alpha$ known to be upregulated in the early stages of liver metastasis. In surgical specimens, activated IGF-IR was observed on HSC-like stromal cells surrounding colorectal carcinoma liver metastases. IGF-targeting in vivo using an IGF-Trap caused a significant reduction in HSC activation in response to metastatic colon cancer cells. Therefore, our data identify IGF as a survival factor for HSC and thereby, a promoter of the pro-metastatic microenvironment in the liver. IGF-targeting could therefore provide a strategy for curtailing the pro-metastatic host response of the liver during the early stages of liver metastasis" [25].

- According Pauli BU et al.: "Many cancers display characteristic organ colonization patterns that do not fit simple, anatomical-mechanical trapping theories of tumor cell dissemination. Organ preferences of metastatic spread appear to be mediated partly by the selective attachment of tumor cells to organ-specific, microvascular endothelium. To study these tumor cell-endothelial cell interactions in an efficient and reproducible manner, we have designed a novel in vitro assay system wherein endothelial cells isolated from large vessels (e.g., aorta) can be modulated to assume phenotypic traits of organ-specific, microvascular endothelium. Modulation is achieved by growing bovine aortic endothelial cells (BAEC) on organ-specific matrix components, termed tumor attachment modulators (TAMs). Using monolayers of modulated BAEC in a tumor attachment assay, we show here that tumor cells which metastasize to a given organ, have a significantly higher binding affinity for BAEC grown on TAMs of the preferred, metastasized organ, than they have for BAEC grown on TAMs of any other organ not colonized by these tumor cells. Lung-metastatic tumor cells (R3230AC-MET, B16-F10) adhere preferentially to BAEC monolayers grown on lung-specific TAMs, whereas liver-metastatic tumor cells (RAW117-H10, M5076) selectively adhere to BAEC grown on liver-specific TAMs. In contrast, nonmetastatic tumors cells (R3230AC-LR, RBTCC-1, 647V) show no such adhesion preferences. Preferential tumor cell adherence is increased by growing BAEC for prolonged periods on organ-specific TAMs. Metastatic preference and organ distribution are mediated, at least in part, by urea-extractable endothelial cell surface components that are regulated by the extracellular matrix" [26].

- Nicolson GL: "The locations of distant secondary tumors in many clinical cancers and animal tumors are nonrandom, and their distributions cannot be explained by simple anatomical or mechanical hypotheses based on the simple lodgment or trapping of tumor cell emboli in the first capillary bed encountered. Evidence from certain experimental tumor systems supports Paget's 'seed and soil' hypothesis on the nonrandom distributions of metastases: the unique properties of particular tumor cells ('seeds') and the different characteristics of organ microenvironment ('soil') collectively determine the organ preference of metastasis. Experimentally, differential tumor cell adhesion to organ-derived microvessel endothelial cells and organ parenchymal cells, differential invasion of basement membranes and organ tissues, and differential responses to organ-derived growth-stimulatory and -inhibitory factors all appear to be important determinants to the organ preference of metastasis. Each tumor may achieve organ specificity because of its own unique set of multiple metastasis-associated properties and responses to host microenvironments. As neoplasms progress to more highly malignant states multisite metastases are more likely and organ-specific metastases may be masked or circumvented owing to stochastic events, tumor cell diversification, host selection processes, and increased production of tumor autocrine molecules that may modulate adhesion, invasion, growth, and other properties important in metastasis" [27].

- Santin AD "Although, to the authors' knowledge, no prospective randomized 
clinical trial has demonstrated improvement in survival following the radical dissection of lymph nodes in the treatment of cancer patients, lymphadenectomy is still routinely performed for curative purposes. For many years, regional lymph nodes (RLNs) in tumor-bearing hosts have been considered anatomic barriers to the systematic dissemination of tumor cells. More recently, the belief has been held that lymph nodes play a completely passive role, by virtue of the observations that many lymphatic and lymphaticovenous shunts bypass RLNs and allow both lymphatic and hematogenous dissemination of malignant cells at an early stage in the vast majority of cancers. Surgical removal of RLNs apparently has no effect, deleterious or beneficial, on the well-being of the host. A literature review to evaluate, from a biologic point of view, the role played by RLNs during the interactions between the tumor and the host's immune system. : in our understanding of the molecular events of antigen recognition by $\mathrm{T}$ cells and T-cell activation have provided strong experimental evidence to demonstrate that these secondary lymphoid organs constitute the primary sites where the specific recognition of tumor antigens and the proper activation of the immune system take place" [28].

- Anna C. Obenauf et al.: "the metastatic cascade involves multiple steps, including invasion, entry into the circulation from the primary tumor, systemic dissemination, arrest and extravasation in secondary organs, settlement into latency, reactivation, outgrowth, and potential seeding of tertiary metastasis. The pattern of affected organs is remarkably variable depending on the cancer type. Some cancer types predominantly spread to one organ (e.g. prostate cancer to bone, pancreatic cancer and uveal melanoma to liver), or show sequential organ specific colonization (e.g. colorectal cancer, CRC, frequently metastasizes first to the liver, later to lungs and brain). Other cancer types, such as breast cancer, lung cancer, or melanoma, are able to colonize many different organ sites, either sequentially or synchronously. While defined organ tropisms are not rigid phenomena, the organ-specific patterns of metastasis are clear. Beyond lymph node spread, the liver, lung, bone and brain are frequently colonized by a variety of cancer types. The skin, ovaries and spleen are less common sites of metastasis. Skin metastases generally occur in melanoma and breast cancer, ovarian metastases in breast and gastric cancers, and spleen metastases almost exclusively in melanoma" [29].

- Gert G. Van den Eynde et al.: "The liver is host to many metastatic cancers, particularly colorectal cancer, the liver is a vital organ, and the extent of its involvement with metastatic disease is a major determinant of survival. Metastatic cells arriving in the liver via the bloodstream encounter the microenvironment of the hepatic sinusoid. The interactions of the tumor cells with hepatic sinusoidal and extrasinusoidal cells (endothelial, Kupffer, stellate, and inflammatory cells) determine their fate. The sinusoidal cells can have a dual role, sometimes fatal to the tumor cells but also facilitatory to their survival and growth. Adhesion molecules participate in these interactions and may affect their outcome. Bone marrow-derived cells and chemokines also play a part in the early battle for survival of the metastases. Once the tumor cells have arrested and survived the initial onslaught, tumors can grow within the liver in 3 patterns, reflecting differing host responses, mechanisms of vascularization, and proteolytic activity. This review aims to present current knowledge of the interactions between the host liver cells and the invading metastases that has implications for the clinical course of the disease and the response to treatment" [30].

- Jenny E. Chu et al.: "Cancer Stem Cells The composition of primary breast tumors has been shown to be heterogeneous with respect to both molecular subtype (luminal A, luminal B, basal-like, HER2-overexpressing, normal breast-like, and claudin-low) and cellular function, even within the same tumor. This heterogeneity can be accounted for by the CSC hypothesis, also known as the hierarchy theory, which posits that there is a small, phenotypically identifiable subpopulation of cancer cells with stem cell-like characteristics. These CSCs sit at the top of this functional hierarchy 
and are postulated to be capable of tumor propagation and maintenance due to their ability to self-renew and to differentiate into the cells comprising the bulk of the tumor. Conversely, the terminally differentiated non-CSCs are not capable of producing large amounts of progeny or of tumor propagation.

Breast CSCs demonstrate an increased metastatic propensity in vitro, in vivo, and in clinical observation. The most common site of breast cancer metastasis is to the bone, but metastatic lesions are also found in the lungs, brain, and liver. The high level of CD44 expression by CSCs has been highlighted as one possible contributor, as both hyaluronan and osteopontin (OPN), common ligands for CD44, are expressed in the bone and other common sites of metastasis, suggesting a possible adhesive interaction for circulating tumor cell arrest. In vitro, the CD44-hyaluronan interaction has been shown to mediate the attachment of metastatic breast cancer cells to human bone marrow endothelial cells . This interaction could be abrogated through the depletion of CD44 expression using RNA interference and induced by the transfection of a CD44low breast cancer cell line with CD44 expression vectors. Breast cancer cell lines exhibit different levels of Chemokine (C-X-C motif) Receptor 4 (CXCR4), which appears to positively correlate with both CSC proportions and the propensity of breast cancer cell lines to metastasize similar observations were made in pancreatic cancer, where the identified CD133+ CSC population, there existed two subpopulations based on CXCR4 expression, only the CXCR4+ population was capable of metastasizing. There is evidence to suggest that CSCs are not only tumor-initiating cells, but also metastasisinitiating cells (M-ICs).

A study of human leukemia revealed that the chemoresistance of leukemic CSCs arises from the quiescent nature of these cells, as they are stationary in the G0 phase, which limits the effectiveness of chemotherapeutics that target actively replicating cells. In humans, an increase in the proportion of CD44+ CD24- breast cancer cells has been observed after neoadjuvant chemotherapy, indicating likely CSC therapy resistance in vivo. Interestingly, BCRP1 is also highly expressed in normal hematopoietic stem cells. The presence and activity of ALDH, an enzyme that is capable of metabolizing and inactivating cytotoxics such as cyclophosphamide, is likely playing a key role in the observed chemoresistance. Other factors potentially prolonging the lifespan of CSCs include the increased expression of antiapoptotic molecules such as Bcl-2 and survivin. It remains unclear whether this observed metastatic ability and resistance to therapy is a property attributable only to the CSCs (i.e., innate therapy resistance), or whether these specialized cells also receive signals from their microenvironment in the secondary organ that enhance their survival and resilience in the face of cytotoxic treatment. New therapeutic targets may therefore emerge as we gain a greater understanding of the organ-specific interactions between tumor cells and secondary organ sites.

CSCs and the Metastatic Microenvironment. Related the origin of the CSC: a CSC may originate from a normal tissue stem cell (SC) that has acquired tumorigenic mutations; or a CSC may originate from a more differentiated progenitor/mature cell that has dedifferentiated and adapted a stem-like phenotype. They demonstrate that a phenotypic equilibrium is consistently reached over time both in vitro and in vivo, although the in vivo growth requires coinjection of basal or luminal cells with irradiated carrier cells to allow for these two subtypes to persist long enough to give rise to stem-like cells. Scaffidi and Misteli successfully generated CSC-like and non-CSClike cells after oncogenic reprogramming of differentiated fibroblasts. They observed a stochastic emergence of a small population of CSC-like cells expressing stage-specific embryonic antigen 1 (SSEA-1), a marker that did not arise in any of their control lines, suggesting that the CSC phenotype may occur spontaneously after the main oncogenic events have occurred . Further work that supports this "dedifferentiation" of non-CSCs into CSCs demonstrates the possibility that IL- 6 may be a key mediator of the process 
and highlights the need for further investigation into the origin of CSCs and the effects of their microenvironment on regulating this cellular plasticity.

Regardless of their origin, the functional similarities between CSCs and normal SCs are striking. Normally, the SC niche provides signals that either maintain SC quiescence, promote symmetrical division leading to self-renewal, or promote asymmetrical division leading to differentiation and progression down the lineage. Interactions between SCs and their niche are highly dynamic and essential for proper function. As SCs depend on the surrounding microenvironment for important signals, it is not unreasonable to hypothesize that CSCs may also rely on their microenvironment to maintain their tumor-initiating and metastasis-initiating capacity and that a "metastatic niche" may exist in those organs in which these cells are more likely to create metastatic lesions. This niche may play an important role in the organ tropism observed in breast and other cancers. Additionally, signals from the metastatic niche may cause the interconversion of non-CSCs that have arrived from the primary tumor into more metastatic CSCs" [31].

- $\quad$ Ciara H. O'Flanagan et al.: "we generated and characterized a metastatic murine TNBC cell line, extending our progression series of TNBC cell lines with shared genetics but differential tumorigenicity and metastatic potential. We identified transcriptomic and metabolic profiles underlying metastatic potential and examined the impact of obesity on metastasis. Our findings show that metastatic cells display altered transcriptomic profiles compared to nonmetastatic cells, including several metabolic genes; metastatic potential is associated with altered bioenergetics, including heightened glycolytic capacity and increased mitochondrial respiration; and obesity increases metastatic potential, causing significant transcriptomic changes, particularly in metabolic pathways. To our knowledge, this is the first study to demonstrate altered metabolic plasticity during progression from nonmetastatic to metastatic TNBC lesion in a model derived from a common genetic background. Our findings suggest that targeting metabolic perturbations associated with metastatic potential is a novel strategy for reducing the burden of metastatic TNBC, particularly in obese women" [32].

According Nicolson GL. "The locations of distant secondary tumors in many clinical cancers and animal tumors are nonrandom, and their distributions cannot be explained by simple anatomical or mechanical hypotheses based on the simple lodgment or trapping of tumor cell emboli in the first capillary bed encountered. Evidence from certain experimental tumor systems supports Paget's 'seed and soil' hypothesis on the nonrandom distributions of metastases, in which the unique properties of particular tumor cells ('seeds') and the different characteristics of each organ microenvironment ('soil') collectively determine the organ preference of metastasis. Experimentally, differential tumor cell adhesion to organ-derived microvessel endothelial cells and organ parenchymal cells, differential invasion of basement membranes and organ tissues, and differential responses to organ-derived growth-stimulatory and -inhibitory factors all appear to be important determinants in explaining the organ preference of metastasis. Each tumor system may achieve organ specificity because of its own unique set of multiple metastasis-associated properties and responses to host microenvironments. As neoplasms progress to more highly malignant states multisite metastases are more likely and organ-specific metastases may be masked or circumvented owing to stochastic events, tumor cell diversification, host selection processes, and increased production of tumor autocrine molecules that may modulate adhesion, invasion, growth, and other properties important in metastasis. The importance of each of these properties, however, appears to vary considerably among different metastatic tumor systems. These and other tumor cell and host properties may eventually be used to predict and explain the unique metastatic distributions of certain human malignancies" [33]. 
- $\quad$ Lu X1, et al.: writed that: "Breast cancer causes mortality by metastasizing to a variety of vital organs, such as bone, lung, brain and liver. Effective therapeutic intervention of this deadly process relies on a better mechanistic understanding of metastasis organotropism. Recent studies have confirmed earlier speculations that metastasis is a non-random process and is dependent on intricate tumor-stroma interactions at the target organ. Both the intrinsic properties of breast cancer cells and the host organ microenvironment are important in determining the efficiency of organ-specific metastasis. Advances in animal modeling, in vivo imaging and functional genomics have accelerated the discovery of important molecular mediators of organspecific metastasis. framework of breast" [34].

- $\quad$ R. R. Langley et al.: "The fact that certain tumors exhibit a predilection for metastasis to specific organs has been recognized for well over a century now. An extensive body of clinical data and experimental research has confirmed Stephen Paget's original "seed and soil" hypothesis that proposed the organ-preference patterns of tumor metastasis are the product of favorable interactions between metastatic tumor cells (the "seed") and their organ microenvironment (the "soil"). Indeed, many of first-line therapeutic regimens currently in use for the treatment of human cancer are designed to target cancer cells (such as chemotherapy) and also to modulate the tumor microenvironment (such as anti-angiogenic therapy). While some types of tumors are capable of forming metastases in virtually every organ in the body, the most frequent target organs of metastasis are bone, brain, liver, and the lung. In this review, we discuss how tumor-stromal interactions influence metastasis in each of these organs" [35].

\section{Discussion}

We have seen in this review some biologic system, taken as models, to extrapolate and example those models to liver role as secondary organ involved in cancer metatstasis process: "Analyzing the different steps of malignant growth (primary tumor, metastasizing tumor cells, secondary tumor), one recognizes an intense interaction between normal and malignant cells. Tumor cells not only induce activities of normal cells, which normally are rarely activated, but also they exploit properties of normal cells for their own purposes. The major mechanisms and processes of this "parasitism" are described in more detail and the results are discussed. Tumors cannot grow beyond a certain size without a supply of blood and lymph vessels by the host (angiogenesis). Metastasizing tumor cells cannot leave the vessel (extravasate) in which they are transported without the cooperation of the respective endothelial cells of the host. An appropriate environment formed by the host tissues is essential for the settlement of tumor cells at secondary sites" [12].

Micorrizza phenomena (eucariotic procariotic evolution, symbiosys, mutual exchange and other) show how relationship can happen in biological systems. Embriogenic development and implant in uterus and migration of macrophages or bone marrow colonizing after a bone marrow transplant can be interesting examples. Properties like migration attitudes of some kind of cells towards gradients or specific receptor. So properties like Colonizating, simbiontic and "incubator role"can be very interesting to be considered. In metastatic process after a stochastic dissemination, some secondary organs seem to display good characteristics to act as hosts for the metastatic cell with an active role in their growth. The liver acts as the SOIL for the metastatic cell as the SEED but added by secondary organ active role in promoting the metastasis process. Fetal liver and its role in hemopoiesis function inspires the great the hint that liver could still maintain properties in facilitating some cellular growth. The same applies to bone tissue which is goes through a great metabolic modification, transitioning from osteoclast, to osteo-blastic cell and turnover. "The Expression of the cell adhesion molecule, E-cadherin, in breast cancer cells can facilitate or even boost breast cancer cell adhesion to hepatocytes for seeding in the liver" [5]. 
The liver microenvironment can induce breast cancer cells to re-express E-cadherin and cause MET. This phenotypic change has the potential to alter cell behavior, and thus may be a critical step for cells to survive at metastatic sites within the liver" [5]. "Liver regeneration is a complex network regulated by various growth factors and cytokines expressed at the site of injury or migrated to the liver via the circulatory system" [14]. "Exposure of carcinoma cells to NPC-conditioned medium isolated soluble factors contributing to outgrowth" [15]. "We recently showed that the simultaneous targeting of glucose and glutamine under calorie restriction could significantly reduce systemic metastatic cancer in the VM-M2 mouse model" [17]. "The liver is a highly metastasis-permissive organ" [20]. "This distinctive biology, notably its hemodynamic features and unique microenvironment, renders the liver intrinsically hospitable to disseminated tumor cells" [20]. "Cancer metastasis is a highly complex process that involves aberrations in gene expression by cancer cells leading to transformation, growth, angiogenesis, invasion, and dissemination, survival in the circulation, and subsequent attachment and growth in the organ of metastasis. Angiogenesis facilitates metastasis formation by providing a mechanism to (1) increase the likelihood of tumor cells entering the blood circulation and (2) provide nutrients and oxygen for growth at the metastatic site" [21].

"HGF is produced by several tissues, including neoplasms; it can therefore provide a stimulus for increased motility of malignant cells by both a paracrine and autocrine mechanism. T" [19]. "Metastasis-associated neutrophils expressed substantially more fibroblast growth factor 2 (FGF2) than naïve neutrophils, indicating neutrophil polarization by the tumor microenvironment" [22]. "In control animals changes to the hepatic microenvironment associated with obesity sustained the presence of tumor cells in the liver and increased the incidence of hepatic metastases after intrasplenic/portal inoculation of colon carcinoma cells" [23]. And we can correspond these regenerative liver properties to: "Hepatic resection remains the primary potentially curative therapeutic modality for liver metastases. The regenerative process that occurs postoperatively is a complex phenomenon, orchestrated by molecular cascades involving growth factors, cytokines, proteolytic enzymes and other proteins. Unfortunately, some of these molecules, such as hepatocyte growth factor, tumour growth factor beta and matrix metallo-proteinases also promote tumour growth and may contribute to the recurrence of liver metastasis" [24].

"Hepatic stellate cells (HSC) play a major role in initiating the liver fibrogenic (wounding) response of the liver and can also orchestrate a pro-metastatic microenvironment in the liver in response to invading cancer cells" [24]. Other factor to be consider is for example "The capacity of the liver to regenerate after severe viral or drug induced hepatitis, or after massive partial hepatectomy, is remarkable" [13], and involved growth factors, some cytokines, including TNF- $\alpha$ and IL-6. "The liver, composed of parenchymal cells-hepatocytes-and non-parenchymal cells including endothelial cells, Kupffer cells, lymphocytes, and stellate cells, has a unique capacity to precisely regulate its growth and mass, which is particularly remarkable since hepatocytes are stable cells and rarely divide in the normal state, as they are quiescent in the G0 phase of the cell cycle. However, their proliferative capacity is initiated in the case of liver tissue loss" [14].

But other insults can induce this process. "NPCs in the metastatic hepatic niche secrete factors that can induce a partial mesenchymal shift in epithelial breast cancer cells thus initiating outgrowth, and that this is in part mediated by EGFR Epidermal growth factor receptor activation" [15]. "The microenvironment must be favorable for tumor cell survival" [17]. "The bone formation and reabsorption release and activate survival and growth promoting factors that may contribute to bone metastases development" [17]. "Epithelial - Mesenchymal transition: Normal cell can lose their epithelial features and acquire mesenchymal characteristics. This process is called 
Epithelial-Mesenchymal Transition, and enables epithelial cells to migrate to a new environment. This occurs mainly during embryogenesis, but in cancer cells this process confers the invasive phenotype" [17]. "Identify IGF as a survival factor for HSC and thereby, a promoter of the pro-metastatic microenvironment in the liver" [25]. "Each tumor system may achieve organ specificity because of its own unique set of multiple metastasis-associated properties and responses to host microenvironments. As neoplasms progress to more highly malignant states multisite metastases are more likely and organ-specific metastases may be masked or circumvented owing to stochastic events, tumor cell diversification, host selection processes, and increased production of tumor autocrine molecules that may modulate adhesion, invasion, growth, and other properties important in metastasis" [27].

"The liver sinusoidal cells can have a dual role, sometimes fatal to the tumor cells but also facilitatory to their survival and growth" [30]. "Breast CSCs demonstrate an increased metastatic propensity in vitro, in vivo, and in clinical observation " [30]. The question is why certain cancer display affinity towards one specific organ and not the others? Is possible that certain factors other than physically, anatomical, logistic, vasculature, capillarity, and lymphatic can also play some role? Is it is even possible that the secondary organ harbors metastatic cells, as an incubation site, a functions could you determine the new home for the migrating metastatic cell, a role that could not played by other organs.

Some biological processes are related to migration properties, growth factor, chemokines, adhesion molecule, neoangiogenesis, metal metallo-proteinsis, tissue trophy properties, microenvironment, gradients, and other autocrine factors? Of course we don't deny altered bio-metabolism, altered gene transcription and other factors may also contribute to the process. Expecially in some secondary organs (incubator?) All of these properties that we speculate, if they come true and proven, must be added to the metabolic role played by liver, regenerative chararacteristic, and also to the fetal hemopoietic function (phenotype experession of its genetic information).

Of course if this roles as incubator comes true, it will be certainly necessary for clinicians to get a deep deep knowledge in this phenomena in order to make it possible to think of new therapeutic strategies to delay if not stop such killing metastatic process. We need to add to our SEED and SOIL model, the active role played by the secondary organ in development metastatic process. Can we consider that in these cases, the liver cell could go back to its fetal periods attaining again some a pitch embryonic Behavior such as its hemopoietic properties? Certainly the speed properties in velocity in cell growth are very comparable to that of the hemopoietic process or to cancer process.

When we analyze the literature, we would find reports related to some biological systems is clear that systems move from an initial status to a final status more energetically efficient. The same also cellular population moves to more usefull condition. The similarity between all these processes may be helpful to show common properties which can be used in therapy? An organ and tissue with such high metabolic reserve (liver) and high turnover (bone) in involving the immune systems relationship (lymph nodes or brain) can be adequate substrates or what we call them incubation environment for metastasis.

\section{Conclusion}

According all this scientific literature is possible to conclude that: In those biologic systems we have given as models, many of biological processes go through a first phase to a final phases which in some cases transitions to a more advantageous, or a sort of parasitism- simbiontic process with advantages to all or what we call it win win mutual benefits situation (organism and parassites). Once we examine all these models, who would extrapolate a similar model for metastasis which we call it the 
"seed and soil" hypothesis: we have different role played by dissemination process of metastatic cells and the secondary organ role. In order to hypothesize and develop new ideas of innovative therapeutic strategies in order to stop, control or the least delay metatstatic process, it is relevant to verify the role played by the secondary organ in boosting metastatic cells inside.

And it's not anymore just an entrapment passive role but we think the evidence shows that there must be active role. So by examining all those evidence, it is incumbent upon us to establish that a simple passive filtration process and interaction are not sufficient to explain many episodes we see and there must be some sort of tissue and organ properties (such as high metabolism, high turnover, high oxygen, availability or some sort of involvement of the immune systems) that could take part in this. What we are suggesting is that there must be something more than passive filtration, there must be an active role in growth vs other organ without this characteristics. Could it be that the metastasis uses a parasite-like - simbiontic exchange? And what could be the consequences in the strategies taken in new treatments?

\section{Etical Considerations}

According this work we can say that related the review the etical consideration are the same used in the original article citated. Related the experimental project: we propose an in vitro experiment under the etical principle For this kind of experiment.

\section{References}

1. Luisetto M, Nili-Ahmadabadi B, Mashori GR, Yesvi A, Sahu RK, et al. Brain and immune system KURU disease a toxicological process?. J Neurosci Neurol Disord. 2018; 2: 014-027. Ref.: https://tinyurl.com/yczxztoe

2. Brundrett MC. Coevolution of roots and mycorrhizas of land plants. New Phytologist. 2012; 154: 275-304. Ref.: https://tinyurl.com/ya29e5du

3. Nance J. Getting to know your neighbor: cell polarization in early embryos. J Cell Biol. 2014; 206 : 823-832. Ref.: https://tinyurl.com/y92xr3km

4. Heckman DS, Geiser DM, Eidell BR, Stauffer RL, Kardos NL, et al. Molecular Evidence for the Early Colonization of Land by Fungi and Plants. Science. 2001; 293: 1129-1133. Ref.: https://tinyurl.com/y78yq243

5. Vogel DY, Heijnen PD, Breur M, de Vries HE, Tool AT, et al. J Neuroinflammation. Macrophages migrate in an activation-dependent manner to chemokines involved in neuroinflammation. $J$ Neuroinflammation. 2014; 11: 23. Ref.: https://tinyurl.com/y9hv87te

6. Martin WF, Garg S, Zimorski V. Endosymbiotic theories for eukaryote origin. Philos Trans R Soc Lond B Biol Sci. 2015; 370: 20140330. Ref.: https://tinyurl.com/y7w7nsb3

7. Fiz F, Marini C, Campi C, Massone AM, Podestà M, et al. Allogeneic cell transplant expands bone marrow distribution by colonizing previously abandoned areas: an FDG PET/CT analysis. Blood. 2015; 125: 4095-4102. Ref.: https://tinyurl.com/y8f2hvmk

8. Ma R, Feng $\mathrm{Y}$, Lin $\mathrm{S}$, Chen J, Lin $\mathrm{H}$, et al. Mechanisms involved in breast cancer liver metastasis. J Transl Med 13: 64. Ref.: https://tinyurl.com/yc8ggnsc

9. Kawada K, Hasegawa S, Murakami T, Itatani $\mathrm{Y}$, Hosogi H, et al. Molecular mechanisms of liver metastasis. Int J Clin Oncol 16: 464-472. Ref.: https://tinyurl.com/ydfrlf8g

10. Desai CS, Khan KM, Ma X, Li H, Wang J, et al. Effect of liver histopathology on islet cell engraftment in the model mimicking autologous islet cell transplantation. 2017; 9: 140-149. Ref.: https://tinyurl.com/y7yaccoh

11. Norkina M, Wingard JR. Recent advances in hematopoietic stem cell transplantation. Version 1 F1000Res. 2017; 6: 870. Ref.: https://tinyurl.com/y8omuzcy

12. Paweletz N, Boxberger HJ. Defined tumor cell-host interactions are necessary for malignant growth Crit Rev Oncog. 1994; 5: 69-105. Ref.: https://tinyurl.com/ybzsdhl7

13. Selden AC, Hodgson HJ. Growth factors and the liver. Gut. 1991; 32: 601-603. Ref.: https://tinyurl.com/ybfbj6d8

14. Tao $\mathrm{Y}$, Wang $\mathrm{M}$, Chen $\mathrm{E}$, Tang $\mathrm{H}$. Liver Regeneration: Analysis of the Main Relevant Signaling Molecules. Mediators Inflamm. 2017; 4256352. Ref.: https://tinyurl.com/y7tyewyv 
15. Taylor DP, Clark A, Wheeler S, Wells A. Hepatic nonparenchymal cells drive metastatic breast cancer outgrowth and partial epithelial to mesenchymal transition. Breast Cancer Res Treat. 2014; 144: 551 560. Ref.: https://tinyurl.com/yaaemcb4

16. Osada S, Matsui S, Komori S, Yamada J, Sanada Y, et al. Effect of hepatocyte growth factor on progression of liver metastasis in colorectal cancer. Hepatogastroenterology. 2010; 57: 76-80. Ref.: https://tinyurl.com/ya5xkq6g

17. Macedo F, Katia Ladeira, Filipa Pinho, Nadine Saraiva, Nuno Bonito, et al. Bone Metastases: An Overview. Oncol Rev. 2017; 11: 321. Ref.: https://tinyurl.com/y9gbljjk

18. Seyfried TN, Huysentruyt LC. On the Origin of Cancer Metastasis. Crit Rev Oncog. 2013; 18: 43-73. Ref.: https://tinyurl.com/yaqupted

19. Jiang WG, Hallett MB, Puntis MC. Hepatocyte growth factor/scatter factor, liver regeneration and cancer metastasis. Br J Surg. 1993; 80: 1368-1373. Ref.: https://tinyurl.com/ycxnj62l

20. Clark AM, Ma B, Taylor LD, Griffith L, Wells A. Liver metastases: Microenvironments and ex-vivo models. Exp Biol Med (Maywood). 2016; 241: 1639-1652. Ref.: https://tinyurl.com/y9jaj2h7

21. Takeda A, Stoeltzing O, Ahmad SA, Reinmuth N, Liu W, et al. Role of angiogenesis in the development and growth of liver metastasis. Ann Surg Oncol 2002; 9: 610-616. Ref.: https://tinyurl.com/y79krfkh

22. Gordon-Weeks AN, Lim SY, Yuzhalin AE, Jones K, Markelc B, et al. Neutrophils promote hepatic metastasis growth through fibroblast growth factor 2-dependent angiogenesis in mice. Hepatology. 2017; 65: 1920-1935. Link: https://tinyurl.com/y96vcq2t

23. Wu Y, Brodt P, Sun H, Mejia W, Novosyadlyy R, et al. Insulin-Like Growth Factor-I Regulates the Liver Microenvironment in Obese Mice and Promotes Liver Metastasis. Cancer Res. 2010; 70: 57-67. Ref.: https://tinyurl.com/y93jdumj

24. Paschos KA, Bird NC. Liver regeneration and its impact on post-hepatectomy metastatic tumour recurrence. Anticancer Res. 2010; 30: 2161-2170. Ref.: https://tinyurl.com/ya9s2thk

25. Fernandez MC, Rayes R, Ham B, Wang N, Bourdeau F, et al. The type I insulin-like growth factor regulates the liver stromal response to metastatic colon carcinoma cells. Oncotarget. 2016; 8: 52281-52293. Ref.: https://tinyurl.com/y7qoyq7o

26. Pauli BU, Lee CL. Organ preference of metastasis. The role of organ-specifically modulated endothelial cells. Lab Invest. 1988; 58: 379-387. Ref.: https://tinyurl.com/yamsbtkr

27. Nicolson GL. Organ specificity of tumor metastasis: role of preferential adhesion, invasion and growth of malignant cells at specific secondary sites. Cancer Metastasis Rev. 1988; 7: 143-188. Ref.: https://tinyurl.com/y7jogjbj

28. Santin AD. Lymph node metastases. Cancer 2000; 88: 175-179. Ref.: https://tinyurl.com/yby6jzfd

29. Obenauf AC, Massagué J. Surviving at a distance: organ specific metastasis. Trends Cancer. 2015; 1: 76-91. Ref.: https://tinyurl.com/y7k74m9e

30. Van den Eynden GG, Majeed AW, Illemann M, Vermeulen PB, Bird NC, et al. The Multifaceted Role of the Microenvironment in Liver Metastasis: Biology and Clinical Implications. 2013; 73: 2031-2043. Ref.: https://tinyurl.com/yafx7snx

31. Che JE, Allan AL. The Role of Cancer Stem Cells in the Organ Tropism of Breast Cancer Metastasis: A Mechanistic Balance between the "Seed" and the "Soil"?. Int J Breast Cancer. 2012; 2012: 209748. Ref.: https://tinyurl.com/y9nl9kan

32. O'Flanagan $\mathrm{CH}$, Rossi EL, McDonell SB, Chen X, Tsai YH, et al. Metabolic reprogramming underlies metastatic potential in an obesity-responsive murine model of metastatic triple negative breast cancer. NPJ Breast Cancer. 2017; 3: 26. Ref.: https://tinyurl.com/ycldhjcl

33. Nicolson GL. Organ specificity of tumor metastasis: role of preferential adhesion, invasion and growth of malignant cells at specific secondary sites. Cancer Metastasis Rev. 1988; 7: 143-188. Ref.: https://tinyurl.com/yda9buax

34. Lu X, Kang Y. Organotropism of breast cancer metastasis. J Mammary Gland Biol Neoplasia. 2007; 12: 153-62. Ref.: https://tinyurl.com/ya4hks3y

35. Langley RR, Fidler IJ. The seed and soil hypothesis revisited - the role of tumor-stroma interactions in metastasis to different organs. Int J Cancer. 2011; 128: 2527-2535. Ref.: https://tinyurl.com/yarg5898

36. Luisetto M. Editorial Efficacy of Oncologic Drug Therapy: Some to rethink in the Management of the System. Journal of Business Management and Economics. 2016; 4. Ref.: https://tinyurl.com/yasrqon9 